\title{
Hydraulic control of continental shelf waves
}

\author{
S. Jamshidi and E. R. Johnson
}

(Received xx; revised xx; accepted xx)

This paper studies the hydraulic control of continental shelf waves using an inviscid barotropic quasi-geostrophic model with piecewise-constant potential vorticity, in which the shelf is represented by a flat step of variable width. A coastal-intensified geostrophic current generates topographic Rossby waves, which can become critical at a local decrease in shelf width when the background current opposes Rossby wave propagation. That is, the shelfbreak perturbation permanently modifies the flow field over arbitrarily large distances and the flow transitions from sub- to supercritical as it crosses the perturbation. Critically-controlled flows also lead to the exchange of significant volumes of water between the shelf and the deep ocean. We derive the boundaries for which critical control occurs in terms of a Froude number and the dimensionless magnitude of the perturbation, and analyse the possible transitions between controlled and far-field flow. When firstorder dispersive terms are included in the model transitions are resolved by dispersive shock waves, which remain attached to the forcing region when the Froude number is close to the boundary for critical flow. Contour dynamic simulations show that the dispersive long-wave model captures the quantitative behaviour of the full quasi-geostrophic system for slowly-varying shelves, and replicates the qualitative behaviour even when the longwave parameter is order one.

\section{Introduction}

Coastal trapped waves (CTWs) are vorticity waves that arise when columns of fluid are forced across isobaths, either by upper-layer Ekman transport or by the interaction of an alongshore current with a change in bathymetry. CTWs are a ubiquitous feature in the worlds oceans and form a major component of the subinertial variability of geostrophic currents. They are extremely long lived, and can communicate the ocean's response to localised events over hundreds to thousands of kilometres. Linear CTWs are governed by a form of the vorticity equation in which the non-dimensional parameter is the slope Burger number (Zhang \& Lentz 2017, eq. 16),

$$
S=\left(\frac{N_{0} H}{f L}\right)^{2},
$$

where $N_{0}$ is a scaling for the buoyancy frequency, $f$ is the Coriolis frequency, and $H$ and $L$ are representative depth and cross-shelf length scales respectively. The slope Burger number illustrates the relative importance of stratification and the continental shelf. For large $S$, the shelf-width scale $L$ is small compared to the Rossby radius of deformation $N_{0} H / f$ and CTWs behave much like Kelvin waves (i.e. they ignore the shelf and propagate as if along a vertical wall). Alternatively when $S \ll 1$ stratification is not important and CTWs are barotropic topographic Rossby waves, often called continental shelf waves (CSWs). CTWs can therefore be thought of as a hybrid between internal Kelvin waves and topographic Rossby waves (Brink 1991). This paper is concerned with CSWs, which are known to occur off the coast of Scotland, along the IcelandFaroe ridge, and on the Amundsen Sea shelf (Gordon \& Huthnance 1987; Miller et al. 
1996; Wåhlin et al. 2016). In particular, we use an idealised quasi-geostrophic model to study the interaction between CSWs and localised changes in bathymetry. We show that when CSWs are generated by a background current flowing counter to Rossbywave propagation the flow can become critically controlled. Zhang \& Lentz (2017) (ZL17 hereafter) have previously shown that this mechanism drives the strong onshore flow that is observed in the Hudson Shelf Valley, and thus critically-controlled flows can lead to an enhanced exchange of shelf and open-ocean water. In this work we employ an inviscid model in which the boundary between shelf and open-ocean water is a material contour that evolves according to a single equation. The model admits a single Rossby mode and is therefore simple enough that we are able to show analytically which regions of parameter space permit hydraulic control, as well as analysing in detail the transition between the controlled state and the far-field flow. An interesting feature of the model is that it admits unsteady solutions where the shelf water penetrates deep into the open ocean, which we call 'offshore plumes'. These occur when the turning flow generated by vortex stretching is weaker than the background coastal current, so that fluid columns which cross the shelfbreak head directly offshore rather than turning rightwards under the image effect.

\subsection{Hydraulic control of continental shelf waves}

CTWs propagate to the right in the Northern hemisphere (facing seawards), and thus can become arrested by a current flowing with the coastline on its left. ZL17 use numerical simulations representative of the Hudson shelf valley to illustrate the asymmetric response of topographically-generated CTWs to the direction of the background wind-driven flow. This is summarised in figure 1, which is adapted from figure 11 of ZL17. Figure 1 is a Hovmöller diagram showing the evolution of sea-surface height (SSH) anomaly, taken in an along-shore slice with fixed off-shore co-ordinate located over the valley. The inset to (a) shows a plan view of the shelf bathymetry, with offshore distance increasing with the ordinate, and the red dashed line shows the location of the Hovmöller slice. The direction of the wind is shown as a thick black arrow in either plot, and is the same as the direction of CTW propagation in (a) and counter to CTW propagation in (b). In either case, a mode-1 CTW propagates away from the valley at early times towards positive $x$. The slope of the grey dashed line gives the speed of the mode-1 CTW, which matches the early-time signal. When the background flow opposes CTW propagation as in (b), a train of standing lee waves develops on the left of the valley and spreads at approximately the mean speed of the background current (the slope of the black dashed line in (b)). ZL17 show that the characteristics of the lee waves are consistent with CTWs that have phase speed equal and opposite to the background flow, and thus they are arrested CTWs. Unlike the higher-mode lee waves, the mode-1 wave (grey dashed line) can escape from the valley because its phase speed is much greater than the mean speed of the background flow. (Note that the mode-1 wave signal leaves the domain after 4 days, whereas the lee wave signal persists near $x=-100$ over the whole simulation.) Martell \& Allen (1979) identify the same response in a simpler barotropic model, and additionally demonstrate that the standing lee waves do not occur in the long-wave limit where the alongshore topographic scale is large compared to the shelf width. The majority of this work is concerned with the case shown in (b), where the background flow opposes CTW propagation.

As noted by Zhang and Lentz, the combination of a wave that propagates away from an obstacle against the background flow and standing waves on the other side is suggestive of hydraulic control, whereby geometric constrictions force a transition from subcritical to supercritical flow (Gill 1977; Johnson \& Clarke 2001; Pratt \& Whitehead 2008). Gill 

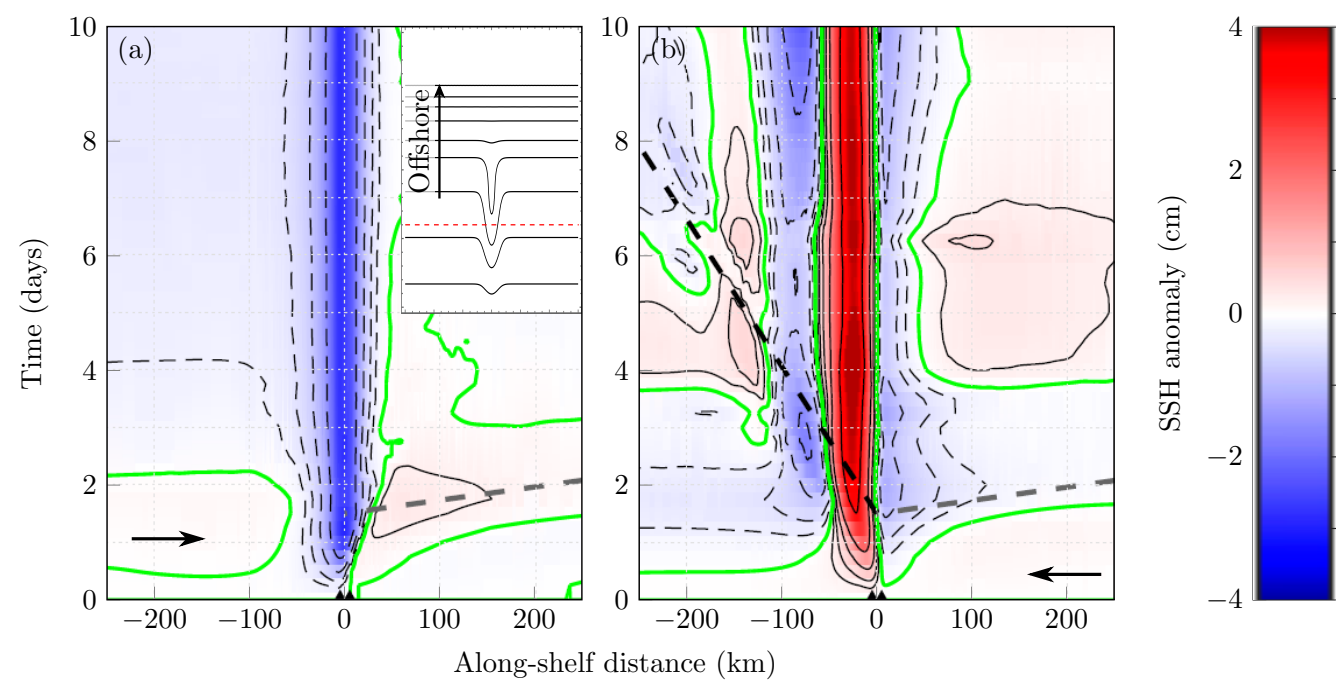

Figure 1: Hovmöller diagram showing sea-surface height (SSH) anomaly in an along-shore slice taken at the centre of the valley. The wind direction is shown as a thick black arrow and drives flow (a) in the direction of CTW propagation, and (b) counter to CTW propagation. The inset to (a) shows a plan view of the bathymetry, with depth increasing offshore. The red dashed line gives the location of the Hovmöller slice. In (a) and (b), the SSH anomaly is defined relative to the flow far away from the valley on the windward side. Thick green contours show curves of zero anomaly. The slope of the grey dashed line is the phase speed of a mode-1 CTW, and the black dashed line in (b) is the mean speed of the background flow. Triangles mark the edge of the valley. Adapted from Zhang \& Lentz (2017), (C) Copyright 2017 AMS.

\& Schumann (1979) and Dale \& Barth (2001) study the hydraulic control of coastal flows using a model where each layer has uniform potential vorticity (PV). This model therefore does not have Rossby waves, and the controlling mode is the internal Kelvin wave $(S \gg 1)$. In contrast, Haynes et al. (1993) study controlled barotropic flow $(S=0)$ in a stepped channel with piecewise-uniform PV and thus a single Rossby mode. They show that two different types of control are possible: one where the flow is controlled at the maximum perturbation in step width, as for Kelvin waves, and one where the flow transitions from one supercritical branch of the solution to another via a control point at the edge of the perturbation. Johnson \& Clarke (1999) extend this model to include firstorder dispersive effects, which enables them to specify the location of the jump between branches. The analytical study of critical control in systems with more than one mode is significantly more complicated; and has been considered by Hughes (1985) and, in the weakly nonlinear limit, by Grimshaw (1987) and Mitsudera \& Grimshaw (1990).

The present work complements previous studies by using an inviscid quasi-geostrophic (QG) model with piecewise-uniform PV to study the control of CSWs generated by a coastal-intensified geostrophic current. By using an idealised model with a single Rossby mode we are able to clearly identify hydraulically-controlled flow, and analytically determine the regions of parameter space in which it occurs in terms of upper and lower bounds on the Froude number, $F$. The idealised model also allows us to study the form of the transition between controlled and far-field flow. In many cases, this transition is accomplished in the long-wave model by a shock (a discontinuity in the off-shore location 
of the material interface separating shelf and ocean water). In the full QG system shocks are replaced by slowly-modulated wave trains, which are the manifestation of standing lee waves in the present model. We analyse these wave trains using the method of 'dispersive shock-fitting' (El 2005; Jamshidi \& Johnson 2020) and show that standing lee waves only occur when $F$ is close to the lower boundary for critical flow.

The rest of the paper is organised as follows. Section 2 develops the model and governing equations, $\S \S 3-4$ analyse the leading and first-order long-wave equations respectively, including conditions for critical control and the form of the transition between controlled and far-field flow. Section 5 compares theoretical results with numerical simulations of both the dispersive long-wave equation and the full QG system, and $\S 6$ discusses the relevance of the present, idealised, model to the real continental shelf.

\section{Model and governing equations}

Consider quasi-geostrophic flow on an $f$-plane, with Cartesian axes Oxyz fixed in a rotating frame of reference. The equation for PV conservation over variable topography $b(x, y)$ is

$$
\frac{\mathrm{D}}{\mathrm{D} t}\left(\nabla^{2} \psi-\frac{\psi}{L_{\mathrm{R}}^{2}}+\frac{f b}{H}\right)=0,
$$

where $L_{\mathrm{R}}=\sqrt{ } g H / f$ is the Rossby radius of deformation, $\psi=g h / f$ is the QG streamfunction which is related to the velocity by $(\partial \psi / \partial x, \partial \psi / \partial y)=(v,-u)$ and $H$ is the mean fluid depth far from the shelf. The conserved quantity in (2.1) is the quasi-geostrophic $\mathrm{PV}$, which we denote $q$. The model is barotropic, although the exact same results apply when a lighter, infinitely-deep, quiescent layer is included in $z>H$ so that we may also choose to interpret the present work as a model for the inviscid dynamics of the bottom layer of the coastal ocean. Fluid occupies the half-plane $y>0$, with a vertical coast at $y=0$ and a flat continental shelf of width $Y_{h}(x)$ which we write

$$
b= \begin{cases}\Pi_{0} H / f & 0<y<Y_{h}(x), \\ 0 & y>Y_{h}(x),\end{cases}
$$

for some $\Pi_{0}>0$. The extension to include a linear continental slope is conceptually straightforward but will not be considered here. We will focus on the case where the shelf-width $Y_{h}$ is a slowly-varying function of $x$, and is constant apart from a localised perturbation around $x=0$. In all numerical simulations that follow we will use

$$
Y_{h}(x)=Y_{0}-\Delta \operatorname{sech}(x / W)^{2},
$$

although the analytic results do not depend on our choice of $\operatorname{sech}(x / W)^{2}$ as the function describing the shelfbreak deviation. Thus the key geometric parameters describing the shelf are $Y_{0}$, the shelf-width away from the perturbation, $\Delta$, the magnitude of the perturbation, and $W$, which measures the width of the topographic forcing region and in the long-wave limit used for analysis is formally large compared to $L_{\mathrm{R}}$. We place no restriction on the magnitude of $\Delta$, but the quasi-geostrophic limit requires that depth variations are small so that application of the present model is best suited to coastlines with a deep continental shelf and $b \ll H$. The present model could also be used to study the dynamics of shelf ridges such as the Charleston bump, in which case $H$ represents the depth of the shelf and $b$ is the height of the ridge, and the inner shelf dynamics are assumed to be isolated from flow at the shelfbreak which is now at $y \rightarrow \infty$. Note also that the inner-shelf interpretation of the model gives a more realistic value for the deformation radius $L_{\mathrm{R}}$, which becomes very large (thousands of kilometres) if the depth 
(a)

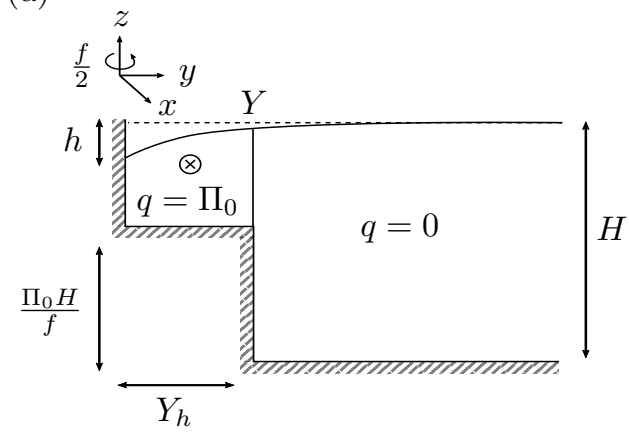

(b)

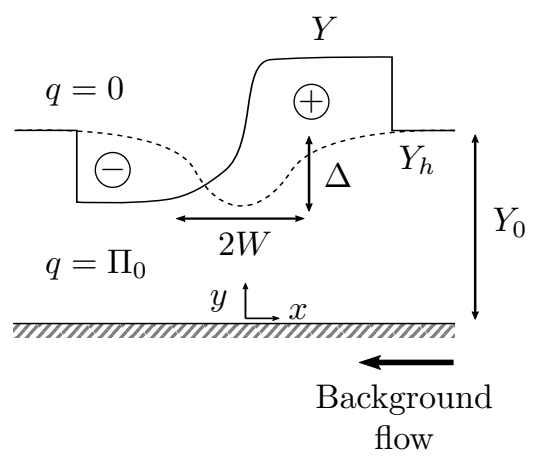

Figure 2: A flat continental shelf occupies the region $0<y<Y_{h}(x)$, with a vertical coast at $y=0$. The model ocean is barotropic, with two regions of uniform PV separated by an interface at $y=Y(x, t)$. Motion is driven by a coastal-intensified background current, in this case from right to left. (a) Side view. (b) Plan view; the dashed curve is $Y_{h}$ and the solid curve is $Y$.

scale $H$ is taken as the mean depth of fluid far from the shelf. Figure 2 shows a schematic of the flow and identifies the various parameters. The long-wave behaviour of the present model without a continental shelf is analysed in detail in Jamshidi \& Johnson (2020) (JJ20 hereafter), and much of what follows here is guided by that analysis.

We will consider the initial-value problem where PV front is initially aligned with the shelfbreak, so that the initial distribution of $\mathrm{PV}$ is

$$
q=\left\{\begin{array}{l}
\Pi_{0} \quad 0<y<Y_{h}(x) \\
0 \quad Y_{h}(x)<y .
\end{array}\right.
$$

The PV is therefore piecewise constant, with a gradient that is entirely due to the topography rather than any internal variation of vorticity, and the model admits a single Rossby wave mode. Models with piecewise-constant PV have been used previously in theoretical studies of coastal outflows (Kubokawa 1991; Johnson et al. 2017) and boundary currents (Pratt \& Stern 1986; Jamshidi \& Johnson 2020), and this restriction is necessary for the analytic work below. The implications of requiring both piecewiseconstant PV and quasi-geostrophic dynamics are discussed further in $\S 6$. Due to the choice of PV profile, the topographic forcing term in (2.1) cancels with $q$ in regions where the $\mathrm{PV}$ front and shelfbreak are aligned so that the governing equation is homogeneous

$$
\nabla^{2} \psi-\frac{\psi}{L_{\mathrm{R}}^{2}}=0 .
$$

With no background flow to disturb the front the unique solution to $(2.5)$ is $\psi \equiv 0$ and the initial condition (2.4) persists for all time. In order to generate CSWs, consider a background flow that starts impulsively at $t=0$, and displaces the PV interface to some $y=Y(x, t)$. In regions of the domain with $Y \neq Y_{h}$, columns of fluid have crossed the shelfbreak and either moved off-shelf and gained relative vorticity $\left(Y>Y_{h}\right.$, plus sign in figure 2) or moved on-shelf and lost relative vorticity ( $Y<Y_{h}$, negative sign). Thus for $y$ between $Y_{h}$ and $Y$ there is a forcing term on the right-hand side of (2.5), the sign of which depends on whether the PV front is on- or off-shelf. Following the algebra through for the various cases, one arrives at the following equation (which is similar to equation 
(2.4) in Haynes et al. (1993)):

$$
\nabla^{2} \psi-\frac{\psi}{L_{\mathrm{R}}^{2}}+\Pi_{0}\left(\mathcal{H}\left(Y_{h}-y\right)-\mathcal{H}(Y-y)\right)=0
$$

where $\mathcal{H}$ is the Heaviside function. The $\mathrm{PV}$ interface $Y(x, t)$ evolves according to the kinematic boundary condition

$$
\frac{\partial Y}{\partial t}=\frac{\mathrm{d}}{\mathrm{d} x} \psi(x, Y(x, t), t),
$$

so that given a closed expression for $\psi(x, Y, t)$ the entire flow field can be tracked by solving the scalar equation (2.7). In writing (2.7) we have assumed that the interface is at all times a single-valued function of $x$. This assumption will later be checked by contour dynamic simulations of the full QG system (2.1) which allow for more complicated interface shapes.

For simplicity we shall restrict discussion to a monotonic, coastal-intensified background flow profile. The appropriate boundary conditions are

$$
\begin{aligned}
\psi=Q_{0} & \text { on } \quad y=0 \\
\psi \rightarrow 0 & \text { as } \quad y \rightarrow \infty
\end{aligned}
$$

along with the requirement that $\psi$ and $u=-\partial \psi / \partial y$ are continuous everywhere. In some oceanographic applications it may be more suitable to choose a background flow that is intensified at the shelf-break, and (2.8a) should be modified accordingly. Note that the system (2.1) and boundary conditions (2.8) are symmetric under the following transformation

$$
\psi \rightarrow-\psi, \quad x \rightarrow-x, \quad b \rightarrow-b, \quad Q_{0} \rightarrow-Q_{0},
$$

so that the problem is equivalent to that of a trench of depth $b$ against a vertical wall.

We non-dimensionalise $\psi$ with $\left|Q_{0}\right|$, horizontal lengths with $L_{\mathrm{R}}$, and introduce $a=L_{\mathrm{R}}\left(\Pi_{0} /\left|Q_{0}\right|\right)^{1 / 2}$. The non-dimensional parameter $a$ is the ratio of the Rossby radius to the vortex length $L_{\mathrm{V}}=\left(\left|Q_{0}\right| / \Pi_{0}\right)^{1 / 2}$, which is the appropriate scale for a vortical current of flux $\left|Q_{0}\right|$ and vorticity $\Pi_{0}$ (Johnson \& McDonald 2006). Alternatively, $a$ measures the relative strengths of the background current and vortical flow driven by columns of fluid crossing the shelfbreak, with large $a$ corresponding to strong vortical flow. Further interpretation of $a$ is given in the context of coastal outflows in Johnson et al. (2017). With these scaling choices, the boundary condition (2.8a) becomes $\psi=Q= \pm 1$ depending on whether the background coastal current is to the right $(Q=+1)$ or the left $(Q=-1)$, and the governing equation is

$$
\nabla^{2} \psi-\psi+a^{2}\left(\mathcal{H}\left(Y_{h}-y\right)-\mathcal{H}(Y-y)\right)=0 .
$$

The key parameters of the problem are thus $a, Q, Y_{0}, \Delta$ and $\epsilon=1 / W$.

\subsection{The long-wave limit}

In the limit $\epsilon=1 / W \rightarrow 0$, we write

$$
\psi(X, y, T)=\psi^{0}+\epsilon^{2} \psi^{1}+O\left(\epsilon^{3}\right) \ldots
$$

where we have introduced the long-wave co-ordinate $X=x / W$ and slow time $T=t / W$. At leading order in $\epsilon$, the field equation (2.10) becomes

$$
\frac{\partial^{2} \psi^{0}}{\partial y^{2}}-\psi^{0}+a^{2}\left(\mathcal{H}\left(Y_{h}-y\right)-\mathcal{H}(Y-y)\right)=0 .
$$


The solution to (2.12) depends on whether the PV front is on the shelf $\left(Y<Y_{h}\right)$ or off the shelf $\left(Y>Y_{h}\right)$. For the case where the front is on the shelf,

$$
\psi^{0}(X, y, T)= \begin{cases}Q \mathrm{e}^{-y}+a^{2} \sinh (y)\left(\mathrm{e}^{-Y}-\mathrm{e}^{-Y_{h}}\right), & 0<y<Y, \\ Q \mathrm{e}^{-y}+a^{2}\left[1-\sinh (y) \mathrm{e}^{-Y_{h}}-\cosh (Y) \mathrm{e}^{-y}\right], & Y<y<Y_{h}, \\ Q \mathrm{e}^{-y}+a^{2}\left(\cosh \left(Y_{h}\right)-\cosh (Y)\right) \mathrm{e}^{-y}, & y>Y_{h},\end{cases}
$$

while when the front is off the shelf,

$$
\psi^{0}(X, y, T)= \begin{cases}Q \mathrm{e}^{-y}+a^{2} \sinh (y)\left(\mathrm{e}^{-Y}-\mathrm{e}^{-Y_{h}}\right), & 0<y<Y_{h}, \\ Q \mathrm{e}^{-y}+a^{2}\left[-1+\sinh (y) \mathrm{e}^{-Y}+\cosh \left(Y_{h}\right) \mathrm{e}^{-y}\right], & Y_{h}<y<Y, \\ Q \mathrm{e}^{-y}+a^{2}\left(\cosh \left(Y_{h}\right)-\cosh (Y)\right) \mathrm{e}^{-y}, & y>Y,\end{cases}
$$

upon enforcing continuity of $\psi$ and $u$ at $y=Y$ and $Y=Y_{h}$ as well as the boundary conditions (2.8). We introduce the index $j=\operatorname{sign}\left(Y_{h}-Y\right)$ to differentiate between the two cases, and write

$$
\begin{aligned}
\psi^{0}(X, Y, T) & =Q \mathrm{e}^{-Y}+\frac{a^{2}}{2}\left[\mathrm{e}^{-\left(Y+Y_{h}\right)}-\mathrm{e}^{-2 Y}+j\left(1-\mathrm{e}^{j\left(Y-Y_{h}\right)}\right)\right] \\
& =Q_{e}\left(Y, Y_{h}\right) .
\end{aligned}
$$

The function $Q_{e}\left(Y, Y_{h}\right)$ depends on $X$ only through location of the PV interface $Y$ and the shelf width $Y_{h}$, and thus is the hydraulic functional for this problem (Gill 1977; Pratt \& Whitehead 2008). The net along-shore flux of shelf water is given by $Q-Q_{e}$. Substituting (2.15) into the kinematic boundary condition (2.7), we have

$$
\frac{\partial Y}{\partial T}+C\left(Y, Y_{h}\right) \frac{\partial Y}{\partial X}=\frac{a^{2}}{2}\left(\mathrm{e}^{j\left(Y-Y_{h}\right)}-\mathrm{e}^{-\left(Y+Y_{h}\right)}\right) \frac{\partial Y_{h}}{\partial X},
$$

which is a forced nonlinear wave equation with long-wave speed

$$
C\left(Y, Y_{h}\right)=-\left.\frac{\partial \psi^{0}}{\partial y}\right|_{y=Y}=Q \mathrm{e}^{-Y}+\frac{a^{2}}{2}\left[\mathrm{e}^{-\left(Y+Y_{h}\right)}-2 \mathrm{e}^{-2 Y}+\mathrm{e}^{j\left(Y-Y_{h}\right)}\right]
$$

Equation (2.16) will be referred to as the hydraulic equation. From left to right, the terms in (2.17) can be identified as the contributions from: background flow, image vorticity due the shelfbreak, image vorticity due to the PV front, and stretching/squashing generated by off- or on-shelf movement of the front. Much of the qualitative behaviour of the hydraulic equation can be understood through $C$. In particular if $Y>Y_{h}$ then $C$ is not a monotonic function of $Y$, but rather has a unique maximum at

$$
Y=Y_{2}=-\log \left(\frac{Q+a^{2} \cosh \left(Y_{h}\right)}{2 a^{2}}\right) \text {. }
$$

Thus, in the language of conservation laws, the flux function $Q_{e}$ may be non-convex when the front is off-shelf. Non-convex flux functions admit a rich variety of compound wave solutions, and are studied in detail for the canonical example of the Riemann problem (i.e. the initial-value problem where the initial condition is a step change in $Y$ ) by El et al. (2017) for the modified Korteweg-de Vries equation, and by JJ20 for the present model without a shelf. Note that (2.18) is valid (i.e. $Y_{2}>Y_{h}$ ) only when $Q<a^{2}$ and

$$
Y_{h}<Y_{2, \mathrm{M}}=\log \left(\frac{\sqrt{\left(1+3 a^{4}\right)}-Q}{a^{2}}\right) .
$$

Thus when $Q=1$ and the background current is in the same direction as CTW phase propagation, compound-wave structures exist when the flow is dominated by vorticity 
$(a>1)$, as in JJ20. However when $Q=-1$ and the background current opposes CTW propagation compound-wave structures exist for all $a$, provided the shelfbreak $Y_{h}$ is sufficiently close to the coast (i.e. $Y_{h}<Y_{2, \mathrm{M}}$ ). Note also that $\partial C / \partial Y$ is discontinuous at the shelfbreak and, for the case where $Q=-1$, changes sign if $Y_{h}>Y_{2, \mathrm{M}}$. Thus compound-wave solutions can also occur in the Riemann problem when the front crosses the shelfbreak; although this situation does not arise in the initial-value problem (2.4).

\subsection{Dispersive effects}

At next order in $\epsilon=1 / W$, the streamfunction correction $\psi^{1}(X, y, T)$ satisfies

$$
\frac{\partial^{2} \psi^{1}}{\partial y^{2}}-\psi^{1}=-\frac{\partial^{2} \psi^{0}}{\partial X^{2}}
$$

Although formally the power series expansion in $\epsilon$ requires that $W \gg L_{\mathrm{R}}$, we show in $\S 5$ that the first-order dispersive correction $\psi^{1}$ captures the qualitative (and much of the quantitative) behaviour of the full quasi-geostrophic system even when $\epsilon=1$ and thus $W=L_{\mathrm{R}}$. This is also true in JJ20 and in long-wave models of coastal outflows (Johnson et al. 2017). Equation (2.20) is to be solved subject to continuity of $\psi^{1}$ and $u^{1}$ at $Y$ and $Y_{h}$, and the coastal boundary condition $\psi^{1}(X, 0, T)=0$. After some algebra, we find:

$$
\begin{aligned}
\psi^{1}(X, Y, T)= & -\frac{a^{2}}{4} \frac{\partial^{2} Y}{\partial X^{2}}+\frac{a^{2}}{4} \mathrm{e}^{-2 Y}\left(\frac{\partial^{2} Y}{\partial X^{2}}-2 Y\left(\frac{\partial Y^{2}}{\partial X}-\frac{\partial^{2} Y}{\partial X^{2}}\right)\right) \\
& +\frac{a^{2}}{4} \mathrm{e}^{-\left(Y+Y_{h}\right)}\left(Y\left(\frac{\mathrm{d} Y_{h}^{2}}{\mathrm{~d} X}-\frac{\mathrm{d}^{2} Y_{h}}{\mathrm{~d} X^{2}}\right)+Y_{h} \frac{\mathrm{d} Y_{h}^{2}}{\mathrm{~d} X}-\frac{\mathrm{d}^{2} Y_{h}}{\mathrm{~d} X^{2}}\left(1+Y_{h}\right)\right) \\
& +\frac{a^{2}}{4} \mathrm{e}^{j\left(Y-Y_{h}\right)}\left(Y\left(\frac{\mathrm{d} Y_{h}^{2}}{\mathrm{~d} X}-j \frac{\mathrm{d}^{2} Y_{h}}{\mathrm{~d} X^{2}}\right)-Y_{h} \frac{\mathrm{d} Y_{h}{ }^{2}}{\mathrm{~d} X}+\frac{\mathrm{d}^{2} Y_{h}}{\mathrm{~d} X^{2}}\left(1+j Y_{h}\right)\right),
\end{aligned}
$$

Substituting (2.21) into the kinematic boundary condition (2.7) gives the dispersive longwave equation

$$
\frac{\partial Y}{\partial T}+\left(C+\epsilon^{2} C^{1}\right) \frac{\partial Y}{\partial X}=\frac{\partial}{\partial X}\left(\psi^{0}(X, Y, T)+\epsilon^{2} \psi^{1}(X, Y, T)\right),
$$

where $C^{1}=-\partial \psi^{1} / \partial Y$ can be computed from (2.21) using

$$
\frac{\mathrm{d}}{\mathrm{d} Y}\left(\frac{\partial^{2} Y}{\partial X^{2}}\right)=\frac{\partial^{3} Y}{\partial X^{3}} / \frac{\partial Y}{\partial X}, \quad \frac{\mathrm{d}}{\mathrm{d} Y}\left(\frac{\partial Y}{\partial X}\right)^{2}=2 \frac{\partial^{2} Y}{\partial X^{2}} .
$$

Note that outside the region of topographic forcing, $Y_{h}$ is constant and (2.21)-(2.22) revert to equations (2.17)-(2.18) in JJ20. An additional conservation law, which is needed for the dispersive shock-fitting applied below, may be formed by multiplying (2.22) through by $Y$ :

$$
\begin{aligned}
\frac{\partial}{\partial T} Y^{2} & +\frac{\partial}{\partial x}\left[2 Q(Y+1) \mathrm{e}^{-Y}\right] \\
& +a^{2} \frac{\partial}{\partial X}\left[(Y+1) \mathrm{e}^{-\left(Y+Y_{h}\right)}-\left(Y+\frac{1}{2}\right) \mathrm{e}^{-2 Y}+(1-j Y) \mathrm{e}^{j\left(Y-Y_{h}\right)}\right] \\
& +a^{2} \epsilon^{2} \frac{\partial}{\partial X}\left[Y^{2} \frac{\partial Y^{2}}{\partial X} \mathrm{e}^{-2 Y}+\frac{1}{4}\left(\frac{\partial Y^{2}}{\partial X}-2 Y \frac{\partial^{2} Y}{\partial X^{2}}\right)\left(-1+(1+2 Y) \mathrm{e}^{-2 Y}\right)\right] \\
& =0 .
\end{aligned}
$$




\section{The hydraulic equation}

In this section we analyse the hydraulic equation (2.16) and show that the range of solutions can be presented in terms of $\Delta$, the shelfbreak perturbation magnitude, and $F$, a Froude number which is defined below.

\subsection{Steady solutions}

By the kinematic boundary condition (2.7), $\psi$ is constant in steady flow. In the hydraulic equation, this requires that

$$
Q_{e}\left(Y, Y_{h}\right)=\Phi
$$

for some constant $\Phi$, with $Q_{e}$ the hydraulic functional defined in (2.15). Contours of $Q_{e}$ for the particular choice of parameters $Q=-1$ (i.e. background flow opposing CTW propagation) and $a=0.8795$ are shown in figure $3(\mathrm{a})$. Given a shelfbreak profile $Y_{h}$ (in fact, given just the far-field width $Y_{0}$ and the perturbation magnitude $\Delta$ ) each contour in figure 3(a) that crosses $Y_{h}=Y_{0}$ and $Y_{h}=Y_{0}-\Delta$ represents a possible steady solution $Y$ to the hydraulic equation (2.16). The steady solution chosen by the initial-value problem can be determined as follows.

For small $\Delta<\Delta_{\text {cr }}$ the flow evolves to become steady in the forcing region, and the steady state is entirely sub- or supercritical. Transient disturbances thus propagate away from the forcing region in one direction only and the initial condition $Y=Y_{0}$ persists on the other side. Thus the constant $\Phi$ may be determined by evaluating (3.1) on the undisturbed side:

$$
\Phi=Q_{e}\left(Y_{0}, Y_{0}\right)=-\mathrm{e}^{-Y_{0}} .
$$

Figure 3(b) shows an example of steady supercritical flow when $Y_{0}=1$ and $\Delta=0.01$. The solid curve is the PV front $Y$ and the dashed curve is the shelfbreak $Y_{h}$, and in order to see the scale of the topographic forcing region this and all other solutions have been plotted in the original co-ordinate $x$. The long-wave co-ordinate $X=x / W$ is found by re-scaling the horizontal axis so that the topographic perturbation lies in $|X|<1$. The contour corresponding to figure 3(b) is highlighted in (a) (upper red dashed curve). The solution starts at $\left(Y, Y_{h}\right)=(1,1)$ and follows the contour $(3.2)$ to the maximum perturbation $Y_{h}=0.99$ as the shelf narrows, before retracing the path to $(1,1)$ as the shelf widens again. The solution is therefore symmetric about the origin and the front is off-shelf $\left(Y>Y_{h}\right)$ throughout.

If $\Delta>\Delta_{\text {cr }}$ is sufficiently large then the contour through $\left(Y_{0}, Y_{0}\right)$ does not extend to the maximum displacement $Y_{h}=Y_{0}-\Delta$ and instead the steady solution selects the unique contour that satisfies

$$
C\left(Y, Y_{0}-\Delta\right)=0
$$

The long-wave speed vanishes at the maximum topographic displacement, which is thus a control point for the flow (Pratt \& Whitehead 2008). The vanishing long-wave speed corresponds to a turning point in the $\left(Y, Y_{h}\right)$-plane, so that the contour selected by the initial-value problem in critically-controlled flow is the one that is horizontal at $Y_{h}=Y_{0}-\Delta$. Given a far-field shelf width $Y_{0}$, the critical value $\Delta_{\text {cr }}$ beyond which the flow becomes controlled is thus that at which the contour through $\left(Y_{0}, Y_{0}\right)$ is horizontal $\left(\Delta_{\text {cr }}\right.$ is shown for $Y_{0}=1$ as a dotted line in figure 3(a)). Figure 3(c) shows an example of critically-controlled flow with $\Delta=0.05$, and the corresponding contour is highlighted in (a) (lower red contour). The solution traces the highlighted section of the contour once, so that $Y$ is monotonic and asymmetric as a function of $Y_{h}$. Note that $Y \neq Y_{0}$ when $Y_{h}=Y_{0}$ so that critically-controlled flow alters the far-field state on both sides of 

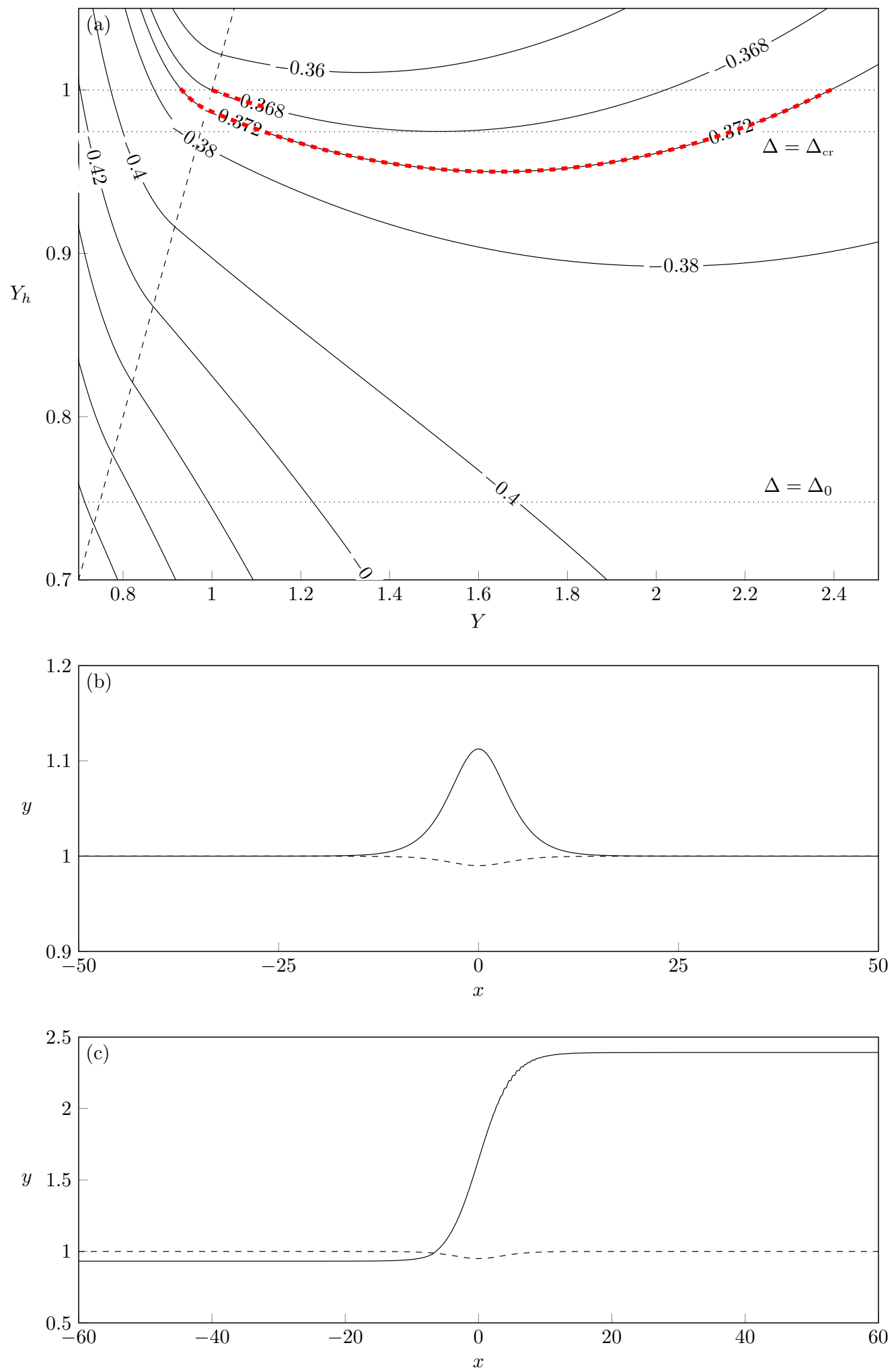

Figure 3: (Continued on the following page) 
Figure 3: (a) Contours of the hydraulic function $Q_{e}\left(Y, Y_{h}\right)$. Steady solutions must lie on a single contour. The black dashed lines are $Y=Y_{h}$ and $Y_{h}=1$, and dotted lines show critical values of the perturbation size $\Delta$ at which the solution changes type when $Y_{0}=1$. The red dashed curves show examples of supercritical and critically-controlled solutions which are plotted as the solid lines in (b) and (c) respectively, and the shelfbreak $Y_{h}(x)$ is shown dashed. The non-dimensional Rossby radius is $a=0.8795$ and $Q=-1$ so that the background flow opposes Rossby wave propagation, with $\Delta=0.01$ in (b) and $\Delta=0.05$ in (c).

the shelfbreak perturbation. Note also that the PV front upstream of the perturbation (relative to the background current, $x>0$ ) has been displaced far into the open ocean, so that the critically-controlled flow is associated with enhanced shelf-open-ocean exchange.

To determine the conditions that lead to critical flow, first consider an asymptotic expansion of the long-wave speed $C\left(Y, Y_{h}\right)$ about the far-field state $Y=Y_{h}=Y_{0}$. To leading order,

$$
C \sim Q \mathrm{e}^{-Y_{0}}-\frac{a^{2}}{2} \mathrm{e}^{-2 Y_{0}}+\frac{a^{2}}{2} .
$$

The right-hand side of (3.4) vanishes when $F=1$, where

$$
F=\frac{-Q}{a^{2} \sinh \left(Y_{0}\right)}
$$

is the Froude number for this problem. The condition $F=1$ can only be satisfied if $Q=-1$ and thus as in ZL17 hydraulic control is only possible when the background current opposes CTW propagation. One can also show that control only occurs when the perturbation is a localised narrowing in shelf width $(\Delta>0)$ as follows. In order for disturbances to propagate away from the forcing region, the critically-controlled solution must have $C>0$ for large positive $X$ and $C<0$ for large negative $X$. Since $C$ is dominated by the the term due to the front moving on- or off the shelf, we can conclude that $Y>Y_{h}$ for large positive $X$ (vortex stretching generates $C>0$ ) and $Y<Y_{h}$ for large negative $X$, so that $\partial Y / \partial X>0$ in controlled flow. Writing the steady version of (2.16) as

$$
C\left(Y, Y_{h}\right) \frac{\partial Y}{\partial X}=\frac{\mathrm{d} Y_{h}}{\mathrm{~d} X} \frac{\partial Q_{e}}{\partial Y_{h}},
$$

where $\partial Q_{e} / \partial Y_{h}>0$, we see that in critically-controlled flow $C$ and $\mathrm{d} Y_{h} / \mathrm{d} X$ have the same sign. That is, $\mathrm{d} Y_{h} / \mathrm{d} X>0$ for $X$ positive and the perturbation must be a local decrease in shelf width. From now on we will restrict our attention to $\Delta>0$ and $Q=-1$ and thus describe $x>0$ as 'upstream' relative to the background flow. Critically-controlled flows are subcritical $(C>0, F<1)$ upstream of the shelfbreak perturbation and supercritical $(C<0, F>1)$ downstream. In completely supercritical flow $C<0$ everywhere, $\mathrm{d} Y_{h} / \mathrm{d} X$ and $\partial Y / \partial X$ have opposite signs and the front is displaced off the shelf (figure 3(b)), while in subcritical flow the front is on the shelf.

Haynes et al. (1993) and Johnson \& Clarke (1999) study the related problem of hydraulically-controlled flow in a stepped channel, and show that several other types of controlled solutions can occur. The fact that these do not appear in the present geometry suggests that they rely on an opposing wall to support their existence, as can be deduced by figure 2 of Johnson \& Clarke (1999). 


\subsection{Offshore plumes}

Assuming that the front is off-shelf at the control point, solving the criticality condition (3.3) gives

$$
Y=-\log \left(\frac{-1}{a^{2}}+\cosh \left(Y_{0}-\Delta\right)\right)
$$

This is the locus of turning points in the hydraulic contours of figure 3(a). As $\Delta \rightarrow \Delta_{0}=Y_{0}-\operatorname{acosh}\left(1 / a^{2}\right)$, the control point $Y \rightarrow \infty$ and the critical solution is no longer valid. The lower dotted line in figure 3 (a) gives $\Delta_{0}$ when $Y_{0}=1$. For $\Delta>\Delta_{0}$ neither the supercritical nor critically-controlled solution exists and the flow never becomes steady. Instead, the flow develops into an ever-expanding 'off-shore plume' similar to the growing solutions for coastal outflow plumes discussed in Johnson et al. (2017) and Jamshidi \& Johnson (2019). As in Johnson et al. (2017), offshore plumes only exist when $a<1$ and the flow induced by vortex stretching as shelf water crosses the shelfbreak is not sufficient to overcome the background current. Instead, the incoming flow is directed principally off shore, and $Y$ grows indefinitely in the forcing region. Offshore plumes have no equivalent in free-surface hydraulic flow, which always becomes steady, but are somewhat related to the 'supercritical leap' of Haynes et al. (1993) in that the flow attains two different supercritical states on either side of the topographic forcing region.

Numerical simulations of (2.16) show that at large times the shape of the front in the source region is approximately constant so that $\partial Y / \partial T$ is independent of $X$. Thus we can obtain an approximate description of the offshore plume through the ansatz

$$
Y(X, T)=Y_{p}(X)+g(T) .
$$

Ignoring terms proportional to $\exp (-2 Y)$ in $(2.16)$ and only considering regions where $Y>Y_{h}$ we have

$$
\frac{\partial Y}{\partial T}=\left[\left(1-a^{2} \cosh \left(Y_{h}\right)\right) \frac{\partial Y}{\partial X}+a^{2} \sinh \left(Y_{h}\right) \frac{\mathrm{d} Y_{h}}{\mathrm{~d} X}\right] \mathrm{e}^{-Y} .
$$

Substituting (3.8) into (3.9) gives the separable equation

$$
\mathrm{e}^{g(T)} \frac{\mathrm{d} g}{\mathrm{~d} T}=\left[\left(1-a^{2} \cosh \left(Y_{h}\right)\right) \frac{\mathrm{d} Y_{p}}{\mathrm{~d} X}+a^{2} \sinh \left(Y_{h}\right) \frac{\mathrm{d} Y_{h}}{\mathrm{~d} X}\right] \mathrm{e}^{-Y_{p}(X)},
$$

where the left-hand side is a function of $T$ alone and the right-hand side is a function of $X$ and so both are equal to $\beta$, a constant. Solving each side separately we find

$$
g(T)=\log \left(T-T_{0}\right)+\log \beta,
$$

and, via the substitution $\exp \left(Y_{p}\right)=\theta(X)\left(-1+a^{2} \cosh \left(Y_{h}\right)\right)$ for $\theta(X)$ unknown,

$$
Y_{p}(X)=\log \left[\left(-1+a^{2} \cosh \left(Y_{h}\right)\right) /\left(X-X_{0}\right)\right]-\log \beta,
$$

so that, at late times in the offshore plume,

$$
Y(X, T) \approx \log \left[\left(-1+a^{2} \cosh \left(Y_{h}\right)\right)\left(T-T_{0}\right) /\left(X-X_{0}\right)\right],
$$

for some constants $T_{0}$ and $X_{0}$. Note that the numerator is an even function of $X$ so that in general (3.12) has two singularities. If $X_{0}>0$ is chosen so that $\cosh \left[Y_{h}\left(X_{0}\right)\right]=1 / a^{2}$, the singularity at $X=X_{0}$ may be eliminated. Equation (3.12) is then valid in $X>-X_{0}$, while for $X<-X_{0}$ the asymptotic solution approaches the unique contour that has its control point at $Y_{h}=\Delta_{0}, Y \rightarrow \infty$. The combined asymptotic solution is singular at $X=-X_{0}$. 


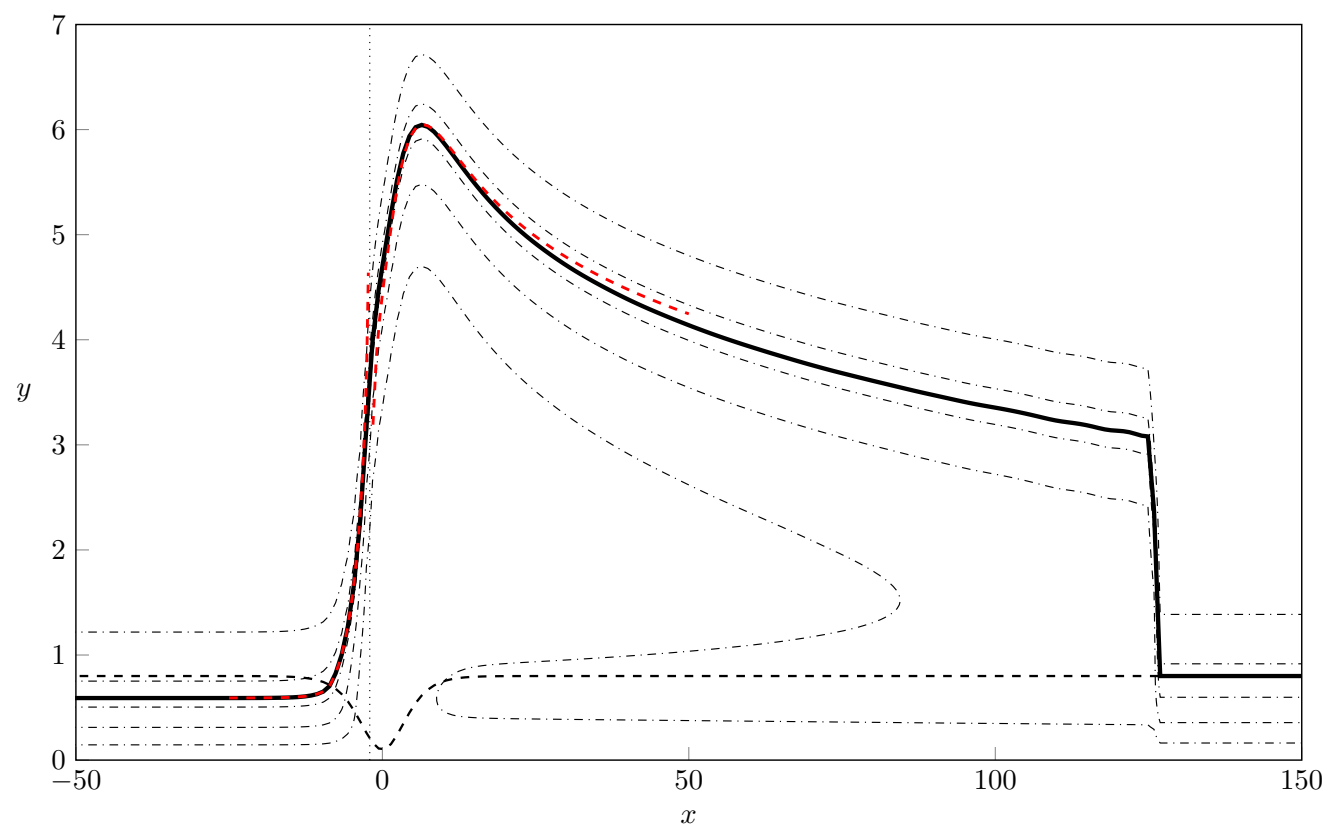

Figure 4: Offshore-plume solution to the hydraulic initial-value problem (2.16).

Dash-dot curves show contours of the streamfunction $\psi^{0}$ at $t=15000$ (contour interval is 0.15 ), the thick black curve is the frontal position $Y$, the red dashed curve is the asymptotic solution (3.12) and the black dashed curve is the topography $Y_{h}(x)$. The black dotted line is $x=-x_{0}$, the location of the singularity in the asymptotic solution. Parameters are $Y_{0}=0.8, \Delta=0.7, a=0.9895$ and $W=5$.

Figure 4 shows instantaneous streamfunction contours in a numerical simulation of the initial-value problem (2.16) in the offshore plume regime. The contours are shown dash-dotted, and the thick black curve is the plume boundary $Y$. There is a slow, broad recirculation of shelf water in the region of topographic forcing and upstream, and the plume boundary at $t=15000$ agrees well with the asymptotic solution (red dashed curve) away from the singularity at $x=-x_{0}$ (the black dotted line). The flow upstream is undisturbed and the alongshore flux of shelf water is $1-\exp \left(-Y_{0}\right)$ while the downstream flux is $1-a^{2} / 2$, which is the asymptotic value of $Q_{e}$ as $Y \rightarrow \infty$.

\subsection{Boundaries for critical control}

An alternative approach to the hydraulic diagram of figure 3 is to consider the problem in the $(\Delta, F)$-plane. Given the far-field shelf-width $Y_{0}$ we seek the range of Froude numbers,

$$
F_{-}(\Delta)<F<F_{+}(\Delta)
$$

for which critical flow occurs. The curves $F_{ \pm}$mark the transition from critical to noncritical flow, and are derived analytically in Appendix A by simultaneously solving the criticality condition (3.3) and the condition for steady, non-critical flow (3.2). These boundaries can also be expressed in terms of the parameter $a$. Figure 5 (a) shows how the $(\Delta, F)$-plane is divided for a narrow shelf $\left(Y_{0}=0.5\right)$. The flow is supercritical for $F>F_{+}$ and subcritical for $F<F_{-}$. For wider shelves (figure 5(b), $Y_{0}=1$ ) the curve $F_{+}(\Delta)$ is non-monotonic and offshore plumes occur in the region marked 'growing', where $F$ lies 

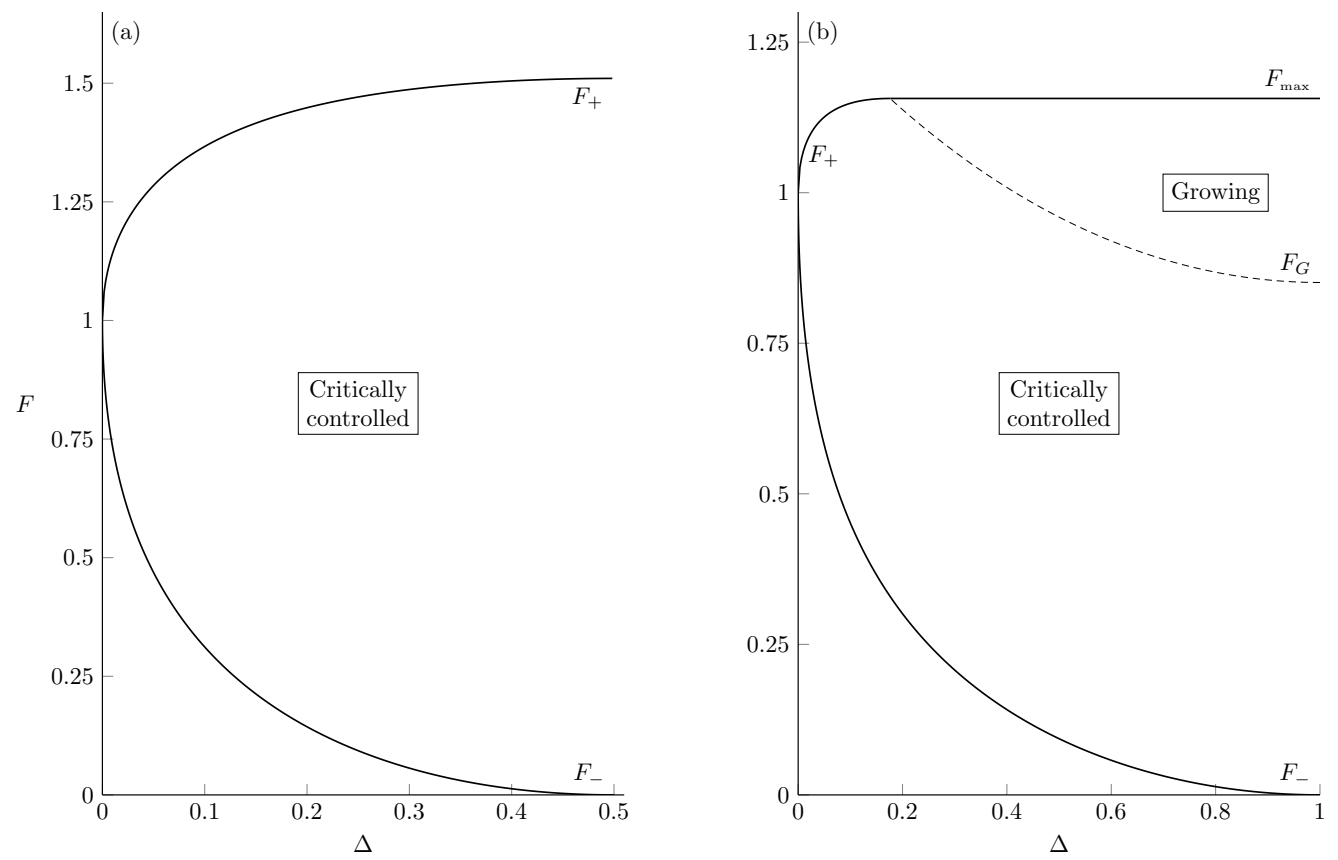

Figure 5: Regions of the $(\Delta, F)$-plane where the flow is critically controlled. (a) A narrow shelf, here with $Y_{0}=0.5$. The flow is critically controlled when $F$ lies between the solid curves $F_{ \pm}(\Delta)$. (b) A wide shelf, here with $Y_{0}=1$. The dashed curve is $F_{\mathrm{G}}(\Delta)$, and offshore plumes occur when $F_{\mathrm{G}}<F<F_{\max }$.

between $F_{\mathrm{G}}(\Delta)$ (shown dashed) and $F_{\max }$, the maximum value of $F_{+}$and also the point of intersection between the two curves.

\subsection{Transition to the far field solution}

Outside the region of topographic forcing, the hydraulically-controlled flow displaces the PV front $Y$ from its initial position $Y_{0}$ to a new, constant, location that we denote $Y_{\mathrm{u} / \mathrm{d}}$ for $x>0$ and $x<0$ respectively. By the heuristic arguments of $\S 3.1$ we expect that controlled solutions are off-shelf upstream and on-shelf downstream, so that $Y_{\mathrm{u}}>Y_{\mathrm{o}}$ and $Y_{\mathrm{d}}<Y_{0}$. If the flow is off-shelf controlled then

$$
\begin{aligned}
\mathrm{e}^{-Y_{\mathrm{u}}} & =-\frac{1}{a^{2}}+\cosh \left(Y_{0}\right) \\
& -\left[\left(\cosh \left(Y_{0}\right)-\cosh \left(Y_{0}-\Delta\right)\right)\left(\cosh \left(Y_{0}\right)+\cosh \left(Y_{0}-\Delta\right)-\frac{2}{a^{2}}\right)\right]^{1 / 2} .
\end{aligned}
$$

To obtain $Y_{\mathrm{u}}$ in on-shelf controlled flow, or $Y_{\mathrm{d}}$ in either case, requires the solution of at least one cubic equation and yields an expression that is too complex to include here. The dependence of $Y_{\mathrm{u} / \mathrm{d}}$ on $F$ and $\Delta$, for the case where $Y_{0}=0.8$, is shown in figure 6 . Increasing $F$ moves the front offshore both downstream and upstream of the topographic perturbation, while increasing $\Delta$ leads to a more extreme displacement of the front relative to the shelfbreak (further offshore upstream, further onshore downstream).

The transition between the topographically-influenced state $Y_{\mathrm{u} / \mathrm{d}}$ and the undisturbed far-field value $Y_{0}$ may be accomplished in one of three ways, depending on the relative value of the long-wave speed $C\left(Y, Y_{0}\right)$ on either side of the transition. Outside of the 

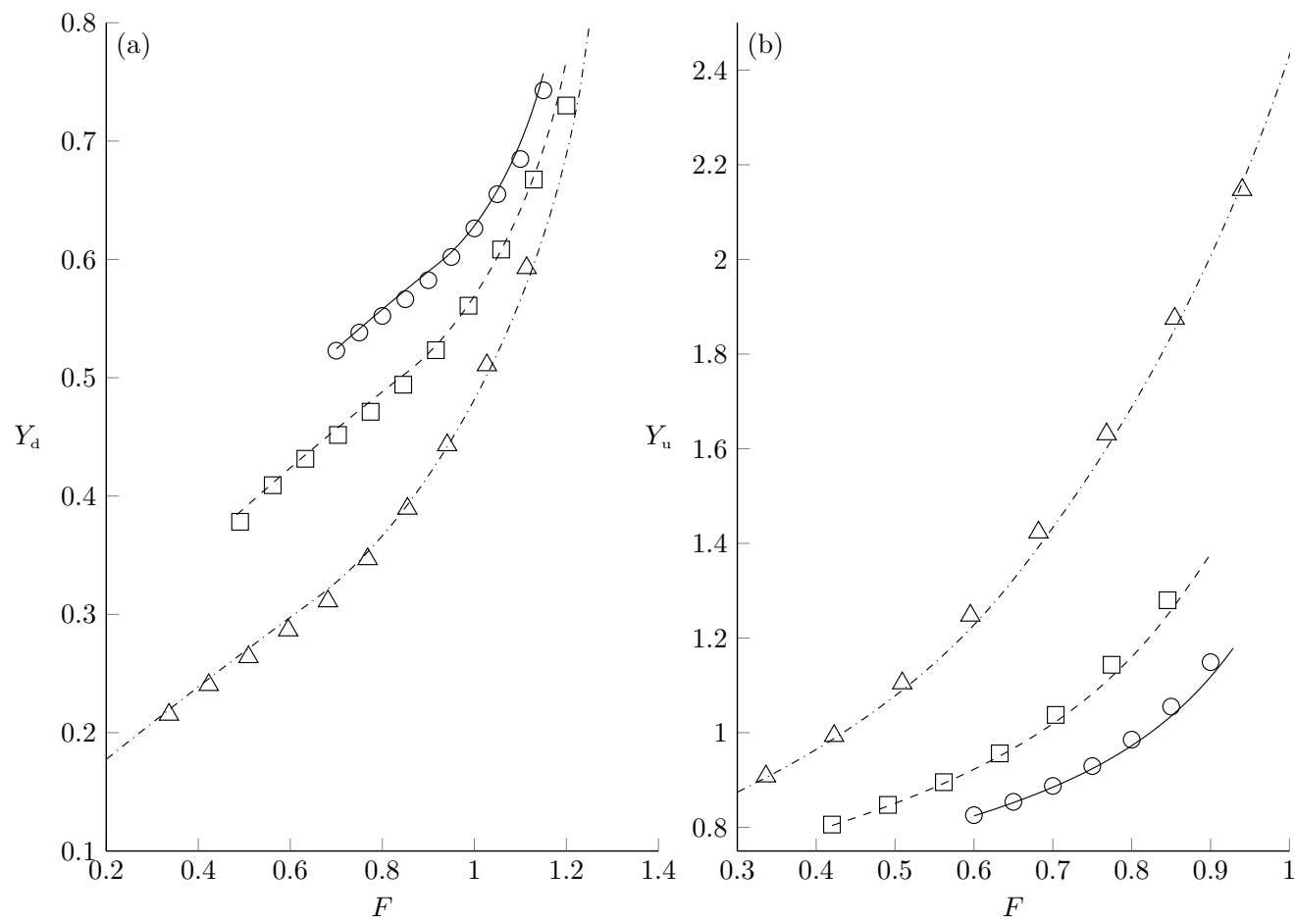

Figure 6: Adjusted frontal position in controlled flow (a) downstream and (b) upstream of the topographic perturbation, for $Y_{0}=0.8$ and various values of Froude number $F$ and topographic perturbation magnitude $\Delta$. Shown are the analytic solutions (curves) and numerical solutions to the dispersive long-wave equation (symbols). The solid curves and circles are for shelves with $\Delta=0.05$, dashed curves and squares with $\Delta=0.1$ and dash-dot curves and triangles with $\Delta=0.25$.

forcing region, characteristic curves in the $(x, t)$-plane are straight lines with slope $\mathrm{d} x / \mathrm{d} t=C\left(Y, Y_{0}\right)$ and so the value of $C$ determines whether curves collide (resulting in a shock) or separate (a rarefaction). The third possibility, a shock-rarefaction, is a compound wave solution that can occur when $C$ is non-monotonic (see JJ20, §3). If the transition from $Y_{0}$ to some value $Y$ is resolved by a shock, this propagates at speed

$$
V(Y)=\frac{Q_{e}\left(Y, Y_{0}\right)-Q_{e}\left(Y_{0}, Y_{0}\right)}{Y_{0}-Y}
$$

First, consider the downstream transition. As noted above, $Y_{\mathrm{d}}<Y_{0}$ and so $C$ is a monotonic increasing function of $Y$. Thus characteristic curves collide and the downstream transition is always resolved by a shock with speed $V\left(Y_{\mathrm{d}}\right)<0$. Next, consider the upstream transition. For $Y>Y_{0}, C$ has a maximum at $Y=Y_{2}$ (given by (2.18) with $Y_{h}=Y_{0}$ ). If $Y_{\mathrm{u}}<Y_{2}, C$ is monotonic increasing and the transition is again resolved by a shock with speed $V\left(Y_{\mathrm{u}}\right)$. Since $Y_{\mathrm{u}}>Y_{0}$ and $V>0$, rearranging (3.14) shows that $Q_{e}\left(Y_{\mathrm{u}}\right)<Q_{e}\left(Y_{0}\right)$ and the transport of shelf water in the controlled solution is reduced compared to the far-field background flow. Thus critical flow 'blocks' the background current by reducing the flow of shelf water towards the topographic perturbation (figure 4 shows that the same occurs in off-shore plumes). If $Y_{0}>Y_{2}$ then $C$ is monotonic decreasing, characteristics separate and the transition is resolved by a rarefaction. This 


\begin{tabular}{c|cccccc} 
Subplot & Type & $F$ & $\Delta$ & $a$ & $t$ \\
\hline & & & & & & \\
(b) & Supercritical & 1.4 & 0.4 & 0.9 & 500 \\
(c) & Offshore plume & 1.2 & 0.6 & 0.97 & 2 & 000 \\
(d) & Shock & 0.9 & 0.1 & 1.12 & 1 & 200 \\
(e) & Shock-rarefaction & 0.9 & 0.4 & 1.12 & 1 & 200 \\
(f) & Subcritical & 0.2 & 0.1 & 2.37 & 40 \\
(g) & Rarefaction & 0.2 & 0.5 & 2.37 & 200
\end{tabular}

Table 1: Details of the different initial value problems displayed in figure 7 . In all cases $Y_{0}=0.8$.

occurs when

$$
F<F_{\mathrm{R}}=\frac{1-3 Z_{0}^{2}}{1-Z_{0}^{2}},
$$

so that, for $F$ sufficiently small and $Z_{0}<1 / \sqrt{ } 3$, critically-controlled flow is resolved upstream by a rarefaction.

In the remaining case, where $Y_{0}<Y_{2}<Y_{\mathrm{u}}, C$ has an interior maximum within the transition and there are three possibilities:

(i) $\quad C\left(Y_{0}, Y_{0}\right)>C\left(Y_{\mathrm{u}}, Y_{0}\right)$. A simple-wave rarefaction cannot connect $Y_{\mathrm{u}}$ and $Y_{0}$ because $C$ has an interior extremum, so the transition is resolved by a shockrarefaction.

(ii) $\quad C\left(Y_{0}, Y_{0}\right)<C\left(Y_{\mathrm{u}}, Y_{0}\right)$ and $V\left(Y_{\mathrm{u}}\right)$ satisfies the Lax entropy condition

$$
C\left(Y_{0}, Y_{0}\right)<V\left(Y_{\mathrm{u}}\right)<C\left(Y_{\mathrm{u}}, Y_{0}\right) .
$$

This ensures that information can propagate into a shock of speed $V\left(Y_{\mathrm{u}}\right)$, and is a necessary condition for such a shock to be physically admissible.

(iii) $\quad C\left(Y_{0}, Y_{0}\right)<C\left(Y_{\mathrm{u}}, Y_{0}\right)$ and $V\left(Y_{\mathrm{u}}\right)>C\left(Y_{\mathrm{u}}, Y_{0}\right)$. In this case, characteristic curves collide but a simple shock does not satisfy the Lax entropy condition and so the transition is resolved by a shock-rarefaction. Following JJ20, the intermediate value $Y_{M}$ at which the shock joins the rarefaction satisfies the equation $C\left(Y_{M}, Y_{0}\right)=V\left(Y_{M}\right)$.

The boundary that determines whether the transition is resolved by a shock or a shockrarefaction can be determined numerically by checking the conditions above. Figure 7 shows representative examples of each type of solution, all with $Y_{0}=0.8$. The solutions are presented in $(\Delta, F)$-space in (a), following figure 5 . The horizontal dotted line is $F_{\mathrm{R}}$, and the dotted curve is the boundary between critical flows that are resolved upstream by a shock, and those resolved by a shock-rarefaction. An example of each type of solution is shown in (b)-(g), again presented in terms of the original variable $x$ so that the scale of the topography is clear. Subplots (d), (e) and (g) are critically controlled, and are all resolved downstream by a shock (not visible in (d) or (e)). Subplot (c) shows an offshore plume, and subplots (b) and (f) are super- and sub-critical flows respectively. The parameters for each run are summarised in table 1. 

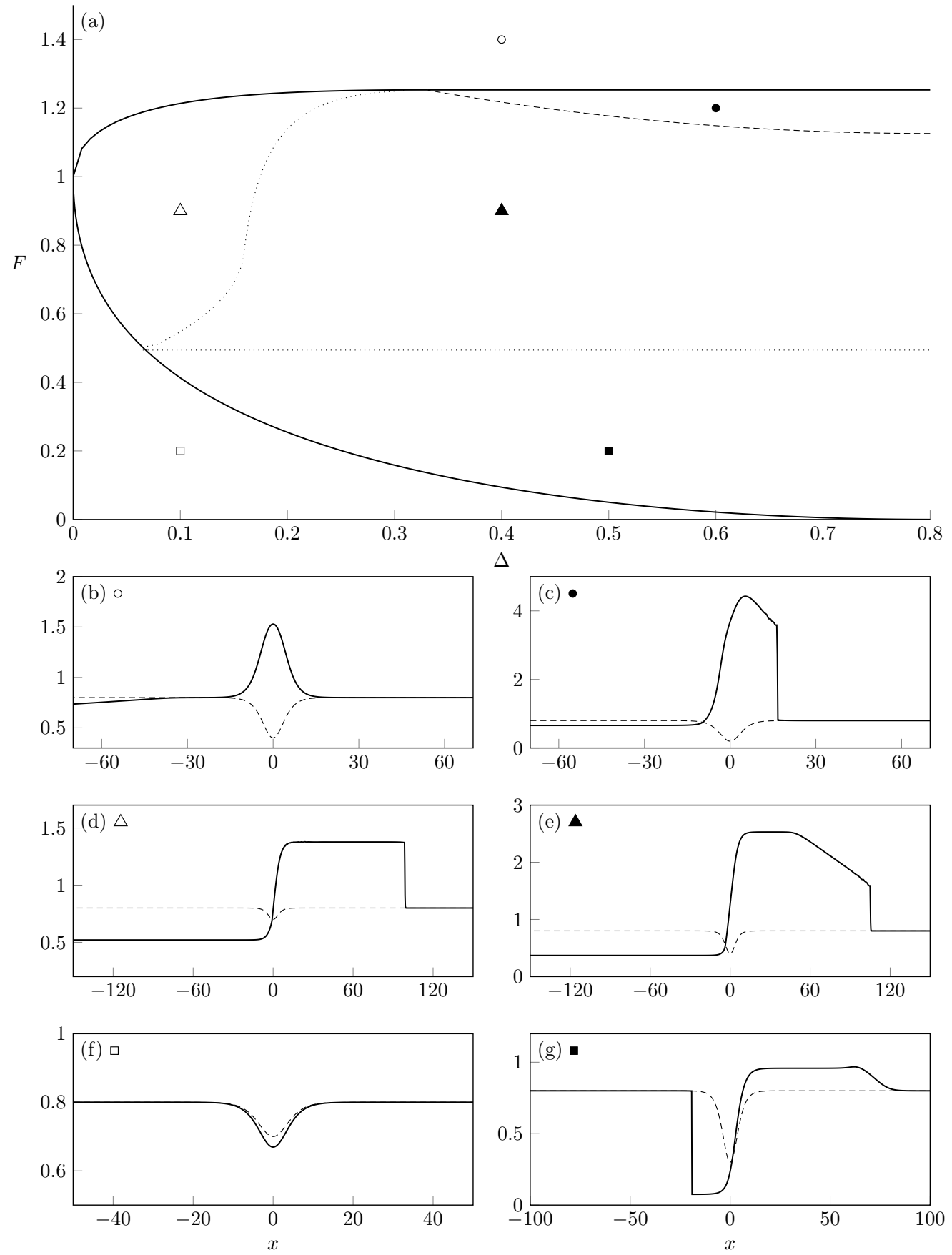

Figure 7: Representative examples of the initial value problem with $Y_{0}=0.8$. (a) Classification of the solution in $(\Delta, F)$-space as in figure 5 . The dotted curves show the boundaries where the upstream transition changes type. (b)-(g) Examples of each type of solution. The black dashed curve is the shelfbreak $Y_{h}(x)$ and the solid curve is the location of the front, $Y(x, t)$. Symbols correspond to the location of the solution in $(\Delta, F)$-space, and full details are given in table 1 . Here and elsewhere solutions are presented in the original variables $x$ and $t$ so that the scale of the topographic forcing is clear. 


\section{The dispersive equation}

The dispersive evolution equation (2.22) may be solved numerically using a pseudospectral method, where the equation is Fourier-transformed in $x$ and advanced in time using an adaptive fourth-order Runge-Kutte scheme. We also employ an artificial damping term at the edge of the domain to allow for longer integration times.

\subsection{Steady solutions}

As in the outflow problem of Johnson et al. (2017), the dispersive initial-value problem selects a different steady solution to that predicted by hydraulic theory. Figure 6 compares analytic solutions from the hydraulic theory (curves) with numerical solutions to the dispersive equation (2.22) (symbols, all computed with $\epsilon=0.2$ ). At this value of $\epsilon$, the difference between the hydraulic predictions for the adjusted far-field state $Y_{\mathrm{u} / \mathrm{d}}$ and the numerically-computed values is small (always less than 10\%, and in most cases much less) so that the hydraulic predictions may be used in the analysis of the dispersive long-wave equation below. However, the differences between the hydraulic and dispersive steady solutions can be resolved by modifying the criticality condition (3.3) to account for the effects of dispersion. Following (2.7) and (2.11), the steady dispersive equation is

$$
\psi^{0}(X, Y)+\epsilon^{2} \psi^{1}(X, Y)=\Phi,
$$

for some constant $\Phi$. Differentiating (4.1) with respect to $X$ gives

$$
\frac{\partial \psi^{0}}{\partial X}+\epsilon^{2} \frac{\partial \psi^{1}}{\partial X}+\frac{\mathrm{d} Y}{\mathrm{~d} X}\left(\frac{\partial \psi^{0}}{\partial Y}+\epsilon^{2} \frac{\partial \psi^{1}}{\partial Y}\right)=0,
$$

where $\partial \psi / \partial X$ vanishes at $X=0$ for symmetric topography (as can be seen by direct computation using (2.15) and (2.21)). Anticipating that $\partial Y / \partial X$ is non-zero at the topographic perturbation in critical flow, the criticality condition for the dispersive equation is

$$
C+\left.\epsilon^{2} C^{1}\right|_{X=0}=0
$$

where $C^{1}=-\partial \psi^{1} / \partial Y$ is a function of $Y_{h}^{\prime \prime}(X)$, so that the dispersive critical solution depends on the curvature of the topographic perturbation at $X=0$ as well as the magnitude of the constriction. By definition, the group velocity is non-zero at the critical section defined by (4.3). Thus in the (time-dependent) dispersive equation, information may propagate away from $X=0$ even in critically-controlled flow. Nevertheless the critical section marks the transition from subcritical to supercritical flow (in terms of the phase speed) and the condition (4.3) determines the unique solution selected by the time-dependent problem (2.22), and indeed by the full QG equations, as can be verified by numerical simulation. (Note that following Grimshaw (1987) one may show that the flows termed 'critical' here are the result of a resonant interaction between free waves and the topographic perturbation. This description may have more physical relevance in the dispersive case where phase and group velocity do not coincide.) Following Johnson \& Clarke (1999), it is simpler to solve the steady equation (4.1) without consideration of (4.3), and verify criticality afterwards. Numerical solutions of (4.1) are computed by truncating the domain at $X= \pm L$ for large $L$, and initially estimating $Y(L)$ as the hydraulic value $Y_{\mathrm{u}}$. This gives an initial guess for $\Phi=\psi^{0}\left(Y_{\mathrm{u}}, Y_{0}\right)$. Equation (4.1) is then integrated from $X=L$ to $X=0$ with the boundary conditions $Y(L)=Y_{\mathrm{u}}$ and $Y^{\prime}(L)=0$, in order to give the subcritical flow and determine $Y(0)$. Since $\Phi$ is known, the supercritical flow in $X<0$ may be found by solving (4.1) as a boundary-value problem using the known value of $Y(0)$ and the boundary condition $Y^{\prime}(-L)=0$. The combined 


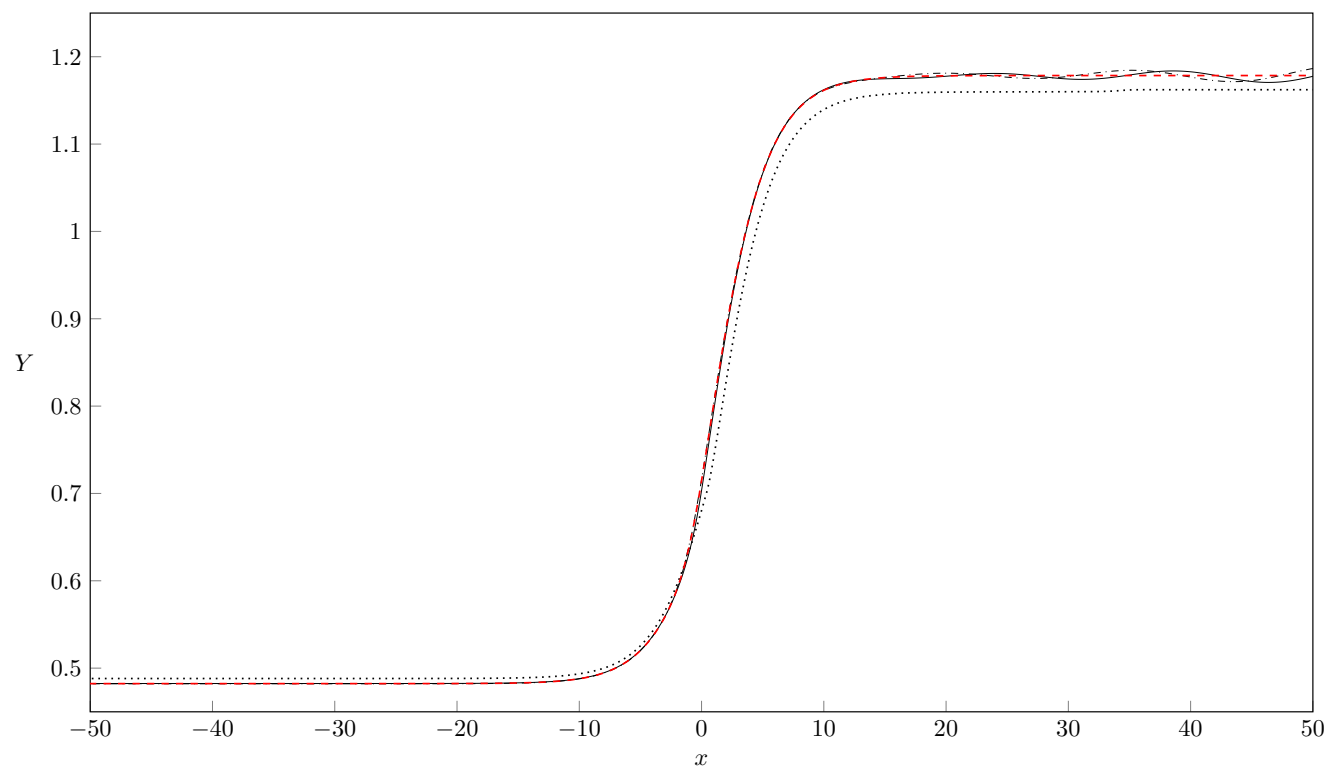

Figure 8: Dispersive critically-controlled solution with $Y_{0}=0.8, \Delta=0.1, F=0.8$ and $\epsilon=0.2$. The solid and dash-dotted black curves show the solution at $t=1000$ for the full QG and dispersive long-wave equations respectively, and the dashed red curve is the numerically computed steady dispersive solution. The critical hydraulic solution is shown dotted for comparison.

solution is necessarily continuous at the origin, but in general $Y^{\prime}$ is discontinuous. The value of $Y(L)$ (and hence $\Phi$ ) is iterated on using Newton's method until $Y^{\prime}(0)$ is continuous. By (2.21), this also enforces continuity of $Y^{\prime \prime}(0)$. Figure 8 shows an example where the upstream hydraulic and dispersive states differ by $2 \%$. The red dashed curve is the critical dispersive solution, while the black curves show numerical integrations of the full QG problem (solid curve) and the long-wave dispersive equation (dash-dotted curve) at $t=1000$. The hydraulic steady solution is shown dotted for comparison. Apart from the presence of small-amplitude waves upstream in the time-dependent solutions all three curves with finite $\epsilon$ are identical, confirming that first-order dispersive effects are sufficient to capture the quantitative behaviour of the QG equation.

\subsection{Transition to the far-field}

In the full QG system, dispersion prevents shocks from forming. Instead, wavesteepening leads to slowly-modulated wave-trains, which are a typical feature of dispersive wave equations (El et al. 2017). For the present work, it is sufficient to note that one common type of modulated wave-train, the dispersive shock-wave or DSW, can be analysed using the method of dispersive shock-fitting (El 2005). DSWs are expanding waveforms with a linear wavetrain at one end and a solitary wave at the other, and are also referred to as undular bores in the context of gravity waves. Dispersive shock-fitting allows one to extract the key parameters of DSWs, namely the wavenumber at the linear end, the conjugate wavenumber (equivalently amplitude) of the solitary wave, and the propagation speed of either end. The same technique also identifies the range of parameters for which transitions are resolved by 'attached DSWs', expanding modulated wave-trains which remain attached to the topographic perturbation much like the 
standing lee waves of Martell \& Allen (1979) and ZL17. A full discussion of DSWs in the flat-bottomed version of the present model is given in JJ20.

\subsubsection{Travelling wave solutions}

We will first set out some basic properties of travelling wave solutions to the dispersive equation, applicable outside the forcing region where $Y_{h} \equiv Y_{0}$ is constant. The dispersion relation for linear waves of wavenumber $k$ propagating on a background $Y_{\infty}$ is

$$
\omega=C\left(Y_{\infty}, Y_{0}\right) k-\mathcal{G}\left(Y_{\infty}\right) k^{3}
$$

where

$$
\mathcal{G}(Y)=\frac{a^{2}}{4}\left[1-\mathrm{e}^{-2 Y}(1+2 Y)\right]
$$

is always positive (c.f. equation 4.2 of JJ20). The soliton dispersion relation is

$$
\widetilde{\omega}=-\mathrm{i} \omega\left(Y_{\infty}, i \widetilde{k}\right)=C\left(Y_{\infty}, Y_{0}\right) \widetilde{k}+\mathcal{G}\left(Y_{\infty}\right) \widetilde{k}^{3},
$$

for $\widetilde{k}$ the half-width of the solitary wave. The fact that the solitary-wave phase velocity can be described by linear-wave dynamics can be seen by considering the exponential tail and making a substitution proportional to $\exp (\widetilde{k} X-\widetilde{\omega} T)$, so that the solitary wave propagates with speed $\widetilde{s}=\widetilde{\omega} / \widetilde{k}$ (Kamchatnov 2019). Comparing soliton and linear phase speeds shows $\widetilde{s}>s$ and so DSWs always have linear waves on the left-hand side.

Writing the dispersive long-wave equation (2.22) in potential form, we have:

$$
\begin{aligned}
\mathcal{G}(Y)\left(\frac{\partial Y}{\partial \xi}\right)^{2}= & a^{2} \mathrm{e}^{-2 Y}+2\left(2-a^{2} \mathrm{e}^{-Y_{0}}\right) \mathrm{e}^{-Y}-2 a^{2} \mathrm{e}^{j\left(Y-Y_{0}\right)} \\
& +4 a^{2} \min \left(Y, Y_{0}\right)+2 s Y^{2}+\alpha Y+E \\
= & \mathcal{V}\left(Y, Y_{0} ; s, \alpha, E\right) .
\end{aligned}
$$

Here $\xi=X-s T$ is a co-ordinate fixed in a reference frame moving with the wave, $s$ is the speed of the travelling wave, and $\alpha$ and $E$ are constants of integration. Note that since $\mathcal{G} \geqslant 0$ travelling-wave solutions to (4.7) exist whenever $\mathcal{V} \geqslant 0$ and we may ignore $\mathcal{G}$ in our analysis. The behaviour of travelling-wave solutions is determined by the number and type of roots of the function $\mathcal{V}$, with solitary waves requiring that $Y_{\infty}$ is a local minimum of $\mathcal{V}$. JJ20 present a full discussion of the range of values of $s$ for which solitary waves exist, but for the present work it is sufficient to note that $C\left(Y_{\infty}, Y_{0}\right)<\widetilde{s}<s_{\mathrm{K}}$, where $s_{\mathrm{K}}$ is the speed of the kink soliton discussed below.

\subsubsection{Compound-wave solutions}

When $Y_{\mathrm{u}}>Y_{2}$ the upstream transition crosses the inflexion point and, as in the hydraulic equation, is resolved by a compound-wave structure which combines a kink soliton with a simple-wave transition (either a modulated wave-train or a rarefaction). The kink soliton is a monotonic travelling-wave solution that connects two far-field states $Y_{\infty}<Y_{2}$ and $Y_{K}>Y_{2}$. In terms of the potential function (4.7), kinks correspond to the case where $\mathcal{V}$ has a double root at both $Y_{\infty}$ and $Y_{K}$ and can thus be thought of as a limiting case of the solitary wave with infinite width (for a finite-width solitary wave, $\mathcal{V}$ crosses the axis at the peak of the wave). Thus kinks can be found by seeking the pair $\left(s_{K}, Y_{K}\right)$ such that $\mathcal{V}\left(Y_{K}\right)=\mathcal{V}^{\prime}\left(Y_{K}\right)=0$, with $\alpha$ and $E$ determined by the requirement that $Y_{\infty}$ is also a double root of $\mathcal{V}$. Since kink solitons are faster than any solitary or linear wave, we expect that they will appear on the right-hand side of any transition that crosses $Y_{2}$ and thus $Y_{\infty}=Y_{0}$. The compound-wave structure is completed by a secondary transition from $Y_{K}$ to $Y_{\mathrm{u}}$. Since $Y_{K}, Y_{\mathrm{u}}>Y_{2}, C$ is monotonic decreasing over 

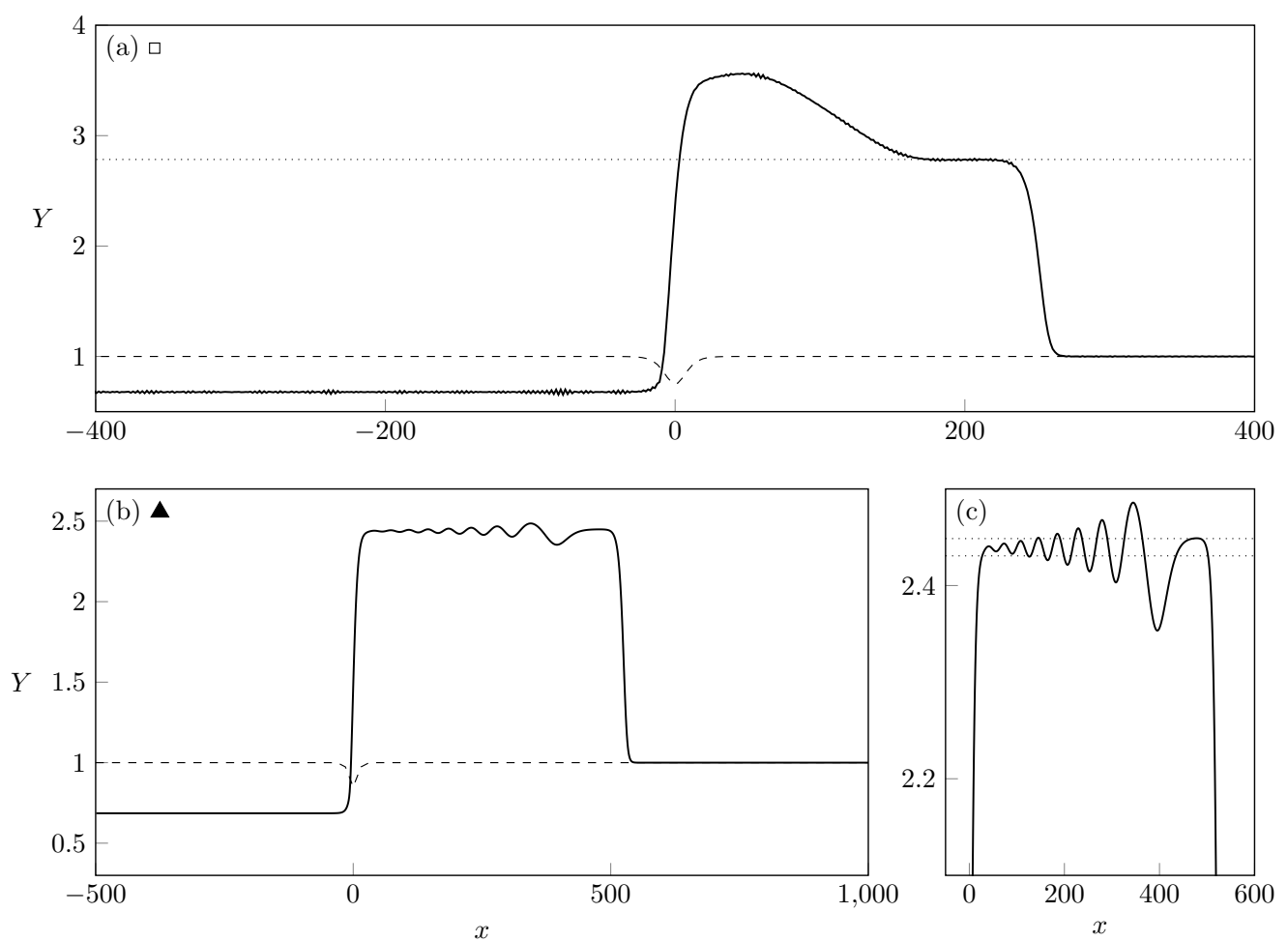

Figure 9: Compound-wave transitions in the dispersive equation. In both cases, $Y_{0}=1$ and $\epsilon=0.1$. (a) The upstream transition is resolved by a $\mathrm{R} \mid \mathrm{K}$. The horizontal dotted line shows $Y_{K}$. (b) The upstream transition is resolved by a $\mathrm{DSW}^{-} \mid \mathrm{K}$. Horizontal dotted lines in the inset (c) show the hydraulic upstream state $Y_{\mathrm{u}}$ and kink level $Y_{K}$. Symbols in (a) and (b) correspond to figure 10(a), which shows the location of the solutions in the $(\Delta, F)$ plane. Full details are given in table 2.

this range and transitions with $Y_{K}<Y_{\mathrm{u}}$ are resolved by a rarefaction-kink (denoted $\mathrm{R} \mid \mathrm{K}$ ). Similarly, transitions with $Y_{K}>Y_{\mathrm{u}}$ are resolved by a depression DSW-kink DSW $^{-} \mid \mathrm{K}$ - the solitary wave is a trough on the background $Y_{\infty}$ ). In general the kink and the simple-wave transition propagate at different speeds, so that at large times the two are separated by a plateau at $Y=Y_{K}$.

A representative example of each type of compound-wave transition is shown in figure 9 . In (a), the transition is resolved by a rarefaction-kink. The kink is at $x \approx 300$, and is connected to the rarefaction by a plateau at $Y=Y_{K}$ (horizontal dotted line). In (b), the kink connects to $Y_{\mathrm{u}}$ via a DSW ${ }^{-}$. For this set of parameters, the difference between $Y_{K}$ and $Y_{\mathrm{u}}$ is small so a zoom of the transition is shown in (c), where the upper and lower horizontal dotted lines show $Y_{K}$ and $Y_{\mathrm{u}}$ respectively.

\subsubsection{Dispersive shock-fitting}

Dispersive shock-fitting is a technique for determining key observables of a DSW (wave number and speed at either end), given the far-field states on either side. For a certain class of equations (integrable equations) the full structure of the DSW arising from the Riemann problem can be written down analytically. El (2005) shows that the key observables may be determined for a much broader class of equations, provided they 


\begin{tabular}{c|ccccccc} 
Subplot & Type & $F$ & $\Delta$ & $a$ & $\epsilon$ & $t$ \\
\hline & & & & & & & \\
$9(\mathrm{a})$ & $\mathrm{R} \mid \mathrm{K}$ & 1 & 0.25 & 0.93 & 0.1 & 10000 \\
$9(\mathrm{~b})$ & $\mathrm{DSW}^{-} \mid \mathrm{K}$ & 0.96 & 0.15 & 0.91 & 0.1 & 16000 \\
$10(\mathrm{~b})$ & Upstream attached & 1 & 0.05 & 0.92 & 0.2 & 10000 \\
$10(\mathrm{c})$ & Both detached & 0.9 & 0.05 & 0.97 & 0.1 & 4500 \\
$10(\mathrm{~d})$ & Downstream attached & 0.75 & 0.03 & 1.07 & 0.2 & 2 & 500
\end{tabular}

Table 2: Details of the initial value problems displayed in figures 9 and 10. In all cases $Y_{0}=1$.

meet a number of technical conditions. These technical conditions are reviewed for the present problem in Appendix B, which also outlines the method for computing the key observables. Assuming that the time taken for the controlled solution to be established over the topography is much less than that required for the full development of a DSW, dispersive shock-fitting may in principle be used to predict the key parameters of DSWs that arise from transitions between critical and far-field flow in the present initial-value problem (El et al. 2009). However we will show below that the downstream solitary wave speed has a local minimum at $F=F_{\text {cr }}$, so that for $F<F_{\text {cr }}$ the downstream wave-train cannot be described using dispersive shock-fitting.

DSWs develop upstream when the hydraulic equation predicts that the transition will be resolved by a simple shock. Thus for a given set of parameters $\left\{Y_{0}, \Delta, F\right\}$ we expect a DSW with $Y_{-}=Y_{\mathrm{u}}$ and $Y_{+}=Y_{0}$, where $Y_{\mathrm{u}}>Y_{0}$ so that the leading solitary wave is one of elevation (i.e. a peak rather than a trough). As discussed in Appendix B, the trailing linear and leading solitary wavenumbers are

$$
\begin{aligned}
k_{\mathrm{u}}^{2} & =\frac{2}{3 \mathcal{G}\left(Y_{\mathrm{u}}\right)^{2 / 3}} \int_{Y_{0}}^{Y_{\mathrm{u}}} \mathcal{G}(Y)^{-1 / 3} \frac{\partial C}{\partial Y} \mathrm{~d} Y, \\
\widetilde{k}_{\mathrm{u}}^{2} & =\frac{2}{3 \mathcal{G}\left(Y_{0}\right)^{2 / 3}} \int_{Y_{0}}^{Y_{\mathrm{u}}} \mathcal{G}(Y)^{-1 / 3} \frac{\partial C}{\partial Y} \mathrm{~d} Y,
\end{aligned}
$$

and the propagation speeds of the upstream DSW edges are

$$
s_{\mathrm{u}}=\left.\frac{\partial \omega}{\partial k}\right|_{Y_{\mathrm{u}}, k_{\mathrm{u}}}, \quad \widetilde{s}_{\mathrm{u}}=\frac{\widetilde{\omega}\left(Y_{0}, \widetilde{k}_{\mathrm{u}}\right)}{\widetilde{k}_{\mathrm{u}}}
$$

respectively. In some cases, $s_{\mathrm{u}}<0$ so that the linear end of the DSW is predicted to enter the region of topographic forcing. Numerical simulations show that in this case the upstream transition is resolved by a partial DSW, which remains attached to the topography and continuously generates waves at the upstream edge of the forcing region. Partial DSWs also occur in free-surface flow over an obstacle, as was shown for the $\mathrm{Su}-$ Gardner (dispersive shallow-water) equations by El et al. (2009). When the upstream transitions is resolved by a $\mathrm{DSW}^{-} \mid \mathrm{K}$ we may apply dispersive shock-fitting to a secondary Riemann problem with $Y_{-}=Y_{\mathrm{u}}$ and $Y_{+}=Y_{K}$ (see $\S 5.2$ of JJ20). 

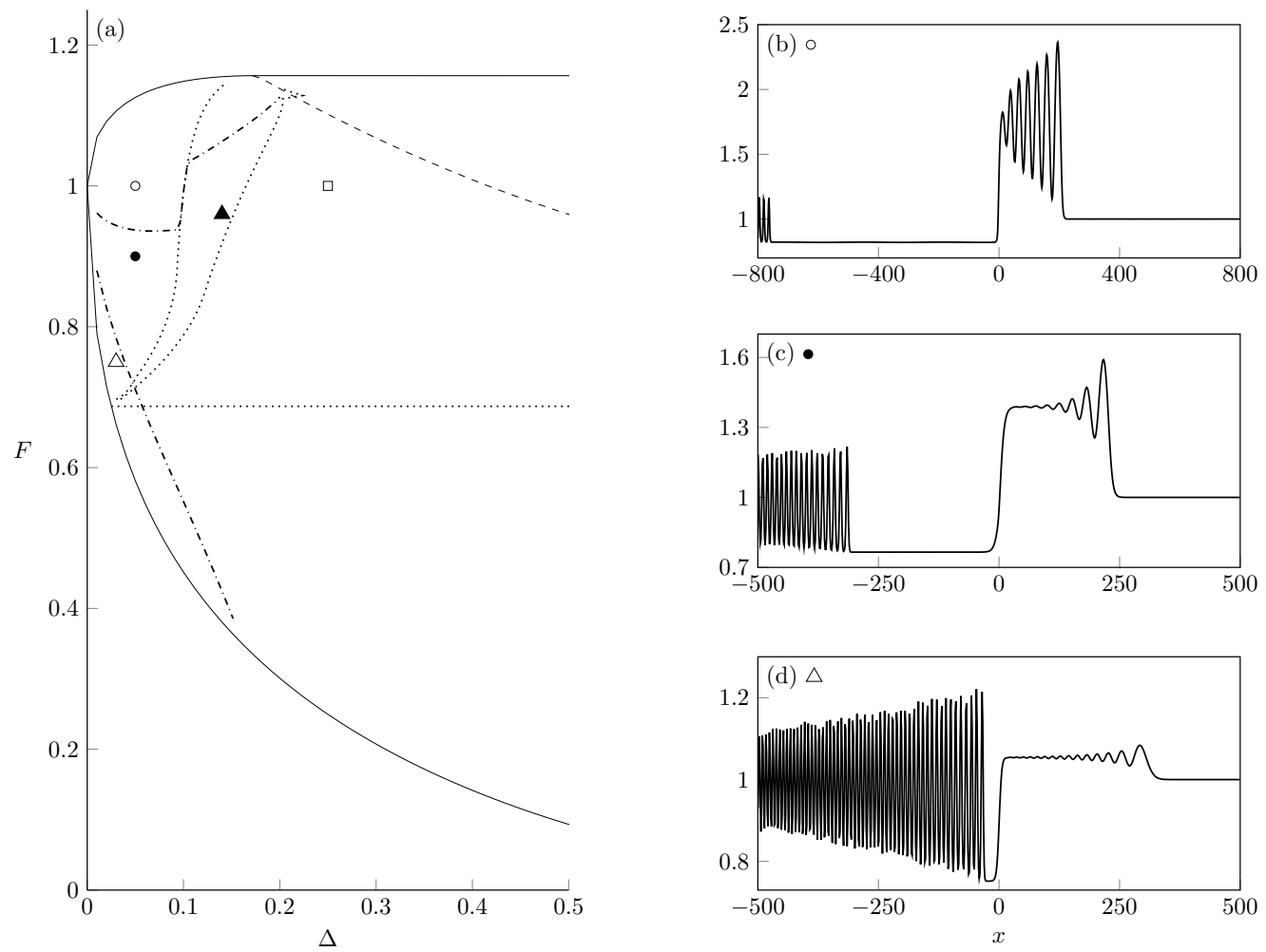

Figure 10: As in figure 7, but for the dispersive equation and with $Y_{0}=1$. The upper and lower dash-dotted curves in (a) mark the boundaries where the upstream and downstream DSWs, respectively, detach from the topography. Dotted curves mark where the upstream transition changes type, from $\mathrm{DSW}^{+}$to $\mathrm{DSW}^{-} \mid \mathrm{K}$ (left-most dotted curve) and then to R|K (right-most dotted curve - see figure 9). Examples of attached and detached DSWs are shown in (b)-(d). Symbols correspond to the location of the solution in $(\Delta, F)$-space, and full details are given in table 2.

Assuming that the downstream transition is resolved by a DSW, we have

$$
\begin{aligned}
k_{\mathrm{d}}^{2} & =\frac{2}{3 \mathcal{G}\left(Y_{0}\right)^{2 / 3}} \int_{Y_{\mathrm{d}}}^{Y_{0}} \mathcal{G}(Y)^{-1 / 3} \frac{\partial C}{\partial Y} \mathrm{~d} Y, \\
\widetilde{k}_{\mathrm{d}}^{2} & =\frac{2}{3 \mathcal{G}\left(Y_{\mathrm{d}}\right)^{2 / 3}} \int_{Y_{\mathrm{d}}}^{Y_{0}} \mathcal{G}(Y)^{-1 / 3} \frac{\partial C}{\partial Y} \mathrm{~d} Y,
\end{aligned}
$$

and the corresponding speeds

$$
s_{\mathrm{d}}=\left.\frac{\partial \omega}{\partial k}\right|_{Y_{0}, k_{\mathrm{d}}}, \quad \widetilde{s}_{\mathrm{d}}=\frac{\widetilde{\omega}\left(Y_{\mathrm{d}}, \widetilde{k}_{\mathrm{d}}\right)}{\widetilde{k}_{\mathrm{d}}} .
$$

The downstream DSW is again one of elevation, with the linear waves on the left. If $\widetilde{s}_{\mathrm{d}}>0$, the downstream DSW remains attached to the topographic perturbation and waves are continuously generated at the downstream edge of the forcing region.

Figure 10 shows a representative example of each type of simple-wave transition (attached downstream DSW, attached upstream DSW, both DSWs detached). The boundaries that divide $(\Delta, F)$-space are plotted as dash-dotted curves in (a), which shows 


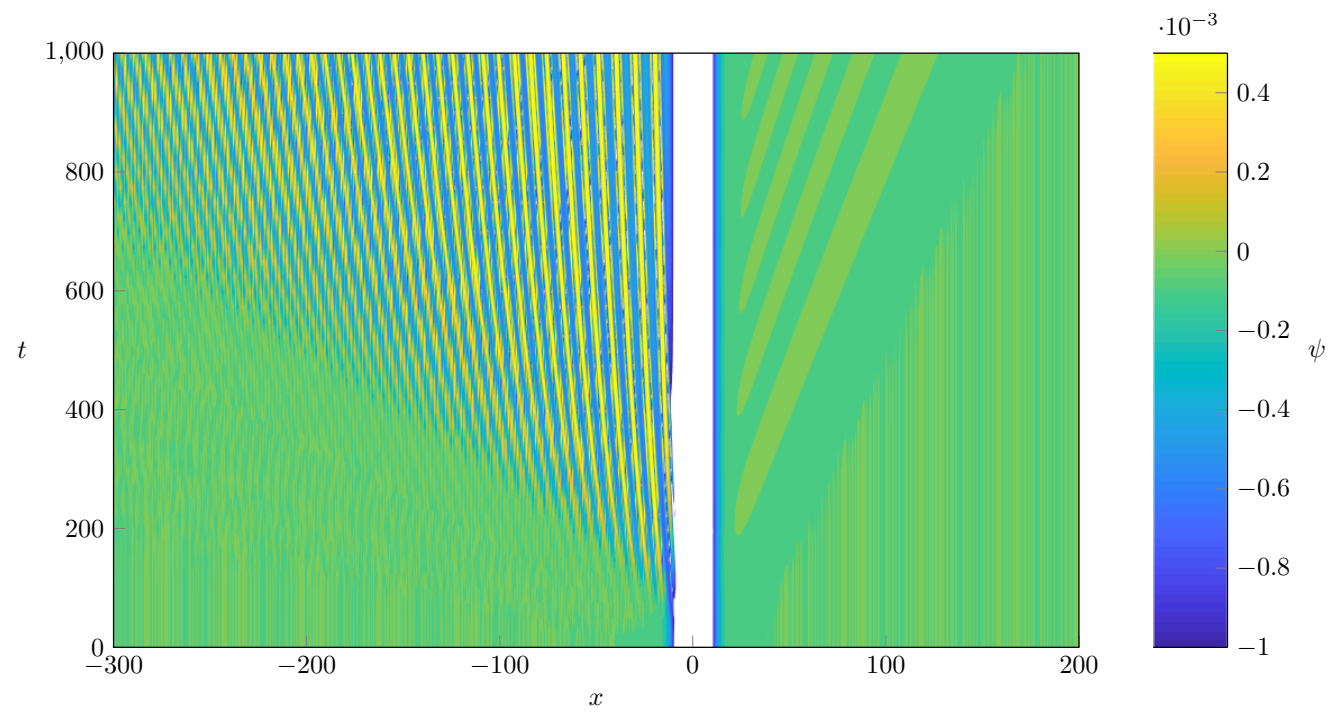

Figure 11: Hovmöller diagram for $\psi$, using the same parameters as figure $10(\mathrm{~d})$ and with $y=Y_{0}-\frac{1}{2} \Delta$. The displacement over the forcing region is an order of magnitude larger, and coloured white for clarity.

that over most of the parameter space both DSWs are detached from the topographic feature as in (c). If $F$ is sufficiently large the upstream DSW can remain attached to the topography as the background current is too strong to allow it to propagate away. An example of this is shown in (b). There is only a small region of parameter space where the downstream DSW remains attached to the topographic feature and in all cases the downstream wave-train spreads much faster than the upstream one, reflecting the fact that the background current and vortex squashing effects reinforce each other in the downstream controlled state. Figure 10(d) shows an example where the downstream DSW is attached, similarly to the standing lee waves of Martell \& Allen (1979) and ZL17. Note that this behaviour only occurs in a small region of parameter space, close to the subcritical boundary $F_{-}(\Delta)$. Figure 11 shows a Hovmöller plot of the streamfunction $\psi$ (equivalently the free surface displacement), taken at the middle of the perturbation with $y=Y_{0}-\frac{1}{2} \Delta$. For clarity we show the free surface anomaly relative to $Q \exp (-y)$ and have coloured the forcing region, where $\psi$ is an order of magnitude larger, white. The standing lee waves develop quickly, while the upstream signal is slower and relatively weaker.

\section{Comparison with numerical results}

\subsection{The dispersive long-wave equation}

Figures 12-13 compare theoretical predictions for kink and solitary wave speed and amplitude with values extracted from numerical integrations of the dispersive long-wave equation (2.22). Due to the difficulties in resolving the linear end of the DSW in numerical simulations, and thus of systematically identifying that end of the wave-train, no attempt was made to validate predictions for the linear wavenumber and group velocity. In all of 

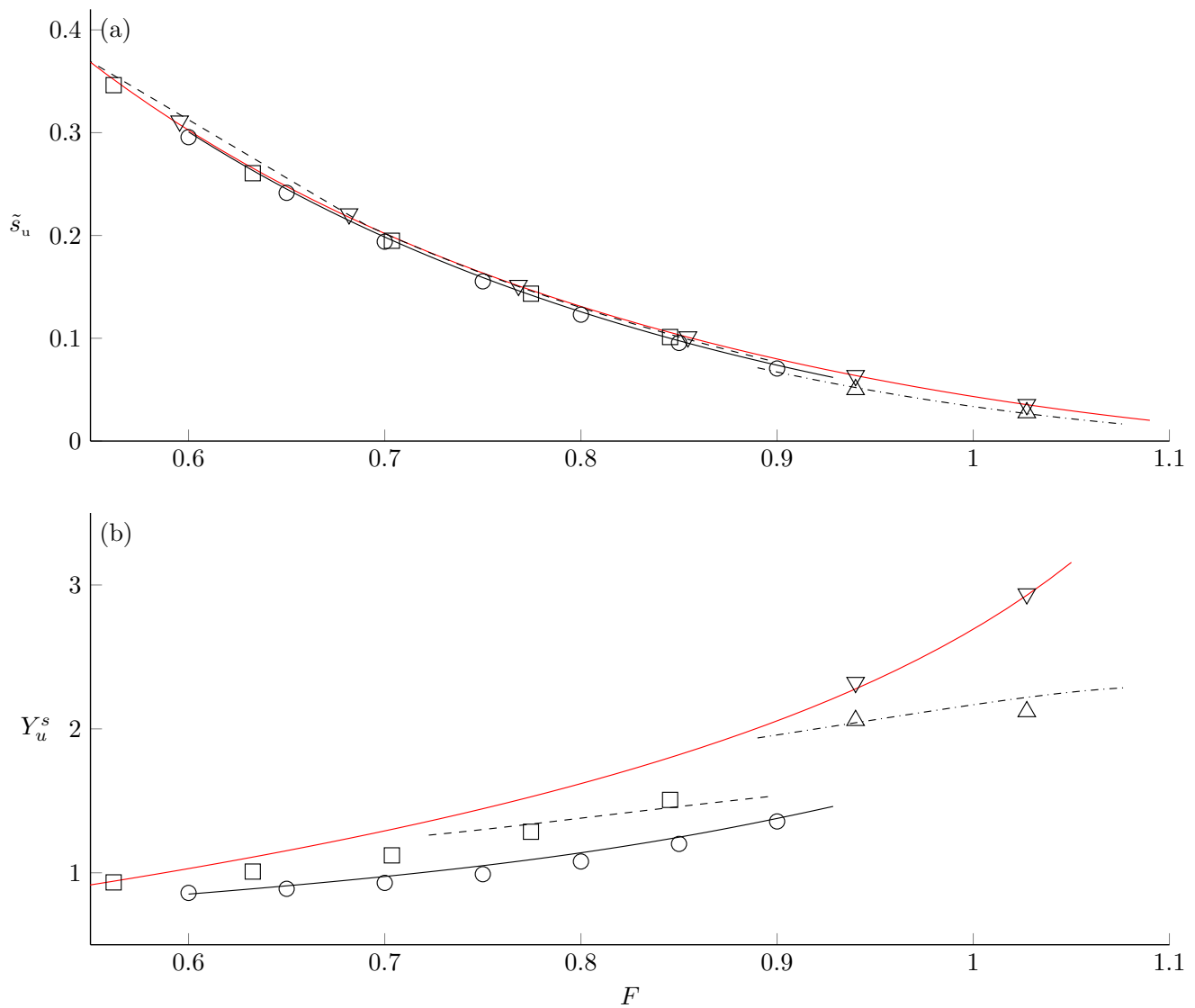

Figure 12: Solitary wave parameters in the upstream DSW, with $Y_{0}=0.8$ and $\epsilon=0.2$. Black curves show the analytical predictions for (a) the speed of the leading solitary wave and (b) the value of $Y$ at the peak of the wave. Curves and symbols are as in figure 6 , with red curves and inverted triangles $(\nabla)$ showing the speed and amplitude of the kink soliton.

the data presented here $Y_{0}=0.8$ and $\epsilon=0.2$, while $F$ was varied across the full critical range for each $\Delta$ to validate the theory for all types of transition.

Figure 12 shows the key parameters of the leading solitary wave in the upstream transition. The speed and amplitude are shown in (a) and (b) respectively, and agreement between theory and numerics is in general very good. The upstream solitary wave speed depends only weakly on $\Delta$, and for all $\Delta$ larger values of $F$ correspond to slower, largeramplitude solitons. The red curve in (a) shows the kink speed $s_{\mathrm{K}}$, which is an upper bound on the solitary wave speed. For $\Delta=0.1$ (dashed curve) and $F<0.7$, dispersive shock-fitting gives an invalid solution with $\widetilde{s}_{\mathrm{u}}>s_{\mathrm{K}}$ and thus amplitude predictions are only shown for $F>0.7$. For $\Delta=0.25$ (dash-dotted curve) all of the upstream transitions considered here are resolved by compound-wave solutions. The inverted triangles $(\nabla)$ in (a) show the kink speed, while those in (b) show the kink amplitude for transitions which are resolved by a $\mathrm{DSW}^{-} \mid \mathrm{K}$. No attempt was made to systematically identify $Y_{K}$ in transitions resolved by a $\mathrm{R} \mid \mathrm{K}$ (those with $F<0.9$ ).

Figure 13 shows (a) the speed and (b) the amplitude of the leading soliton in the downstream transition. For $\Delta=0.05$ and 0.1 (solid curve and circles, dashed curve and 

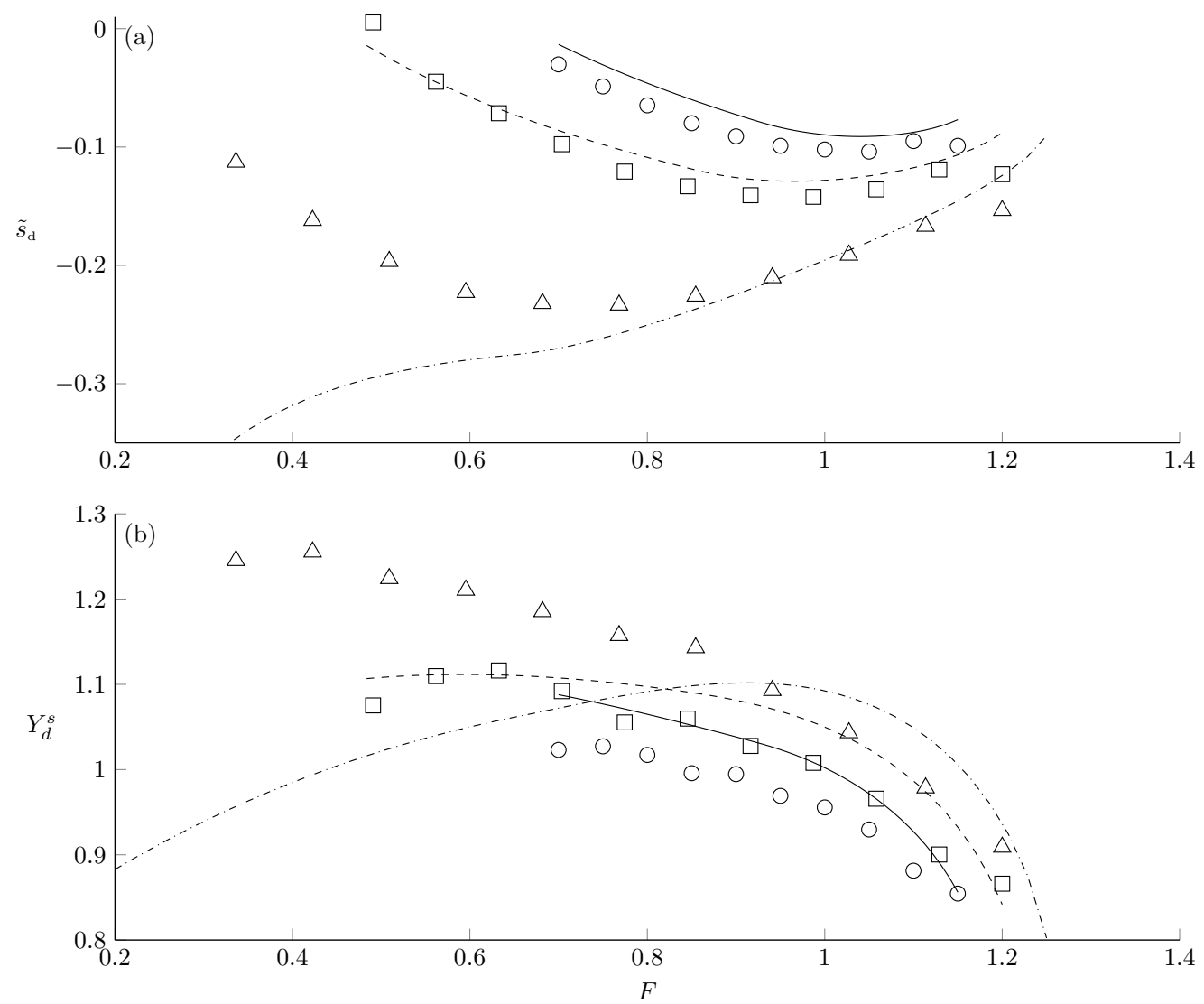

Figure 13: As in figure 12, but for the downstream solitary wave.

squares respectively) agreement between theory and numerics is reasonable. Dispersive shock-fitting describes the DSW in the limit $t \rightarrow \infty$, and at the time when integration was stopped the amplitude of the downstream solitary wave was increasing slowly. It is expected that longer integrations would reduce the error in (b) in cases where the amplitude is less than the predicted value. However in some cases the amplitude is greater than the predicted value, and indeed the numerical results for $\Delta=0.25$ (dash-dot curve and triangles) are qualitatively different from the theory. This may be due to the apparent minima in $\widetilde{s}_{\mathrm{d}}$ at $F=F_{\mathrm{cr}}(\Delta)$ seen in both the theory and numerics in (a). El et al. (2006) analyse the modulation equations for the $\mathrm{Su}-\mathrm{Gardner}$ system and show that a minimum in $s$ as a function of the initial jump amplitude in the Riemann problem corresponds to linear degeneracy in the Whitham system. Numerical simulations show that the DSW terminates at the point of degeneracy, and the linear end is replaced by a finite-amplitude wavefront (their figure 7). As the initial jump amplitude increases beyond the critical value (which corresponds to $F<F_{\text {cr }}$ in figure 13(a)) the terminal point of the DSW moves closer to the solitary-wave end. The parameters (4.8)-(4.11) are derived assuming that the DSW is fully formed, and thus dispersive shock-fitting cannot formally be used when $F<F_{\text {cr. }}$. 


\subsection{Quasi-geostrophic equations}

The fully nonlinear free-boundary QG system (2.1) can be solved numerically to a high level of accuracy using the method of contour dynamics (CD) with surgery (Dritschel 1988). We performed several simulations based on an adaptation of Dritschel's algorithm. The appropriate Green's function for (2.1) is the modified Bessel function $K_{0}\left(\sqrt{ } x^{2}+y^{2}\right)$, and topography is accounted for by including a contour in $0<y<Y_{h}(x)$ with negative vorticity. To account for the wall, we must also include image contours at $y=-Y$ and $y=-Y_{h}$.

Figure 14 compares CD simulations of the full QG problem with predictions from the dispersive long-wave model for critically-controlled flow. Red dashed curves show the dispersive controlled solution computed as in $\S 4.1$, which agrees excellently with the solution to the full problem over the forcing region. The horizontal dotted line in (a) is the amplitude prediction for the upstream leading solitary wave, and is greater than the maximum amplitude obtained in the CD simulation. In fact in the CD simulation the amplitude of the leading wave reaches a maximum value around $t=200$ and then decreases slowly from there, suggesting that higher-order dispersion smooths the upstream transition and reduces the amplitude of the solitary wave. The maximum peak observed in the CD simulation is 1.089 , while dispersive shock-fitting predicts an amplitude of 1.099. Thus the discrepancies are small enough that dispersive shock-fitting may be used to estimate the speed of the leading solitary wave - the analytical prediction is $\widetilde{s}_{\mathrm{u}}=0.128$ while the average speed of the leading peak over $200<t<900$ in the CD simulation is 0.120 . For the parameters used in (a) $\widetilde{s}_{\mathrm{d}}>0$ so that the downstream DSW is attached to the topography, and indeed the CD simulation shows that a modulated wave-train develops on the downstream side of the forcing region but does not propagate away. Long-wave theory may be used to predict the size of the largest wave: the stationary solitary wave on the background $Y_{\infty}=Y_{\mathrm{d}}$ has its crest at $Y_{\mathrm{d}}^{s}=1.25$, while at $t=900$ in the CD integrations the crest of the largest wave is at $Y=1.26$.

In (b), both DSWs are detached from the topography. However for this set of parameters $F<F_{\text {cr }}$ so that the downstream DSW is partially degenerate and its properties cannot be predicted using dispersive shock-fitting. Indeed, the amplitude of the leading wave in the downstream DSW is greater than the prediction obtained using dispersive shock-fitting (bottom horizontal dotted line). The theory again appears to underpredict the amplitude of the leading wave upstream (upper dotted line), although in this case the amplitude was still increasing when the integration was halted at $t=1000$. The analytical prediction for the speed is $\widetilde{s}_{\mathrm{u}}=0.129$ while in the CD simulation $s=0.127$ when averaged over $750<t<1000$. In (c), the upstream DSW is attached to the topography. Here, $F>F_{\text {cr }}$ and the dispersive long-wave theory accurately predicts the amplitude of the solitary wave that leads the downstream DSW.

Figure 14(d) shows a simulation with $\epsilon=1$ and thus is a check on the validity of the long-wave theory. At this extreme value of $\epsilon$ the dispersive long-wave theory does not accurately predict the adjusted values $Y_{\mathrm{u} / \mathrm{d}}$, but the difference is still less than $5 \%$. In fact the contour dynamic simulation lies between the hydraulic and dispersive long-wave predictions in the source region, which suggests that the departure from hydraulic theory is not a monotonic function of $\epsilon$. However the qualitative behaviour is much the same, with a monotonic steady solution across the source region and dispersive wave-trains up- and downstream. The difference between the dispersive controlled solution and that selected by the CD simulation is greater upstream, and correspondingly the prediction for $Y_{\mathrm{u}}^{s}$ is better than that for $Y_{\mathrm{d}}^{s}$. Further CD simulations (not shown) suggest that 

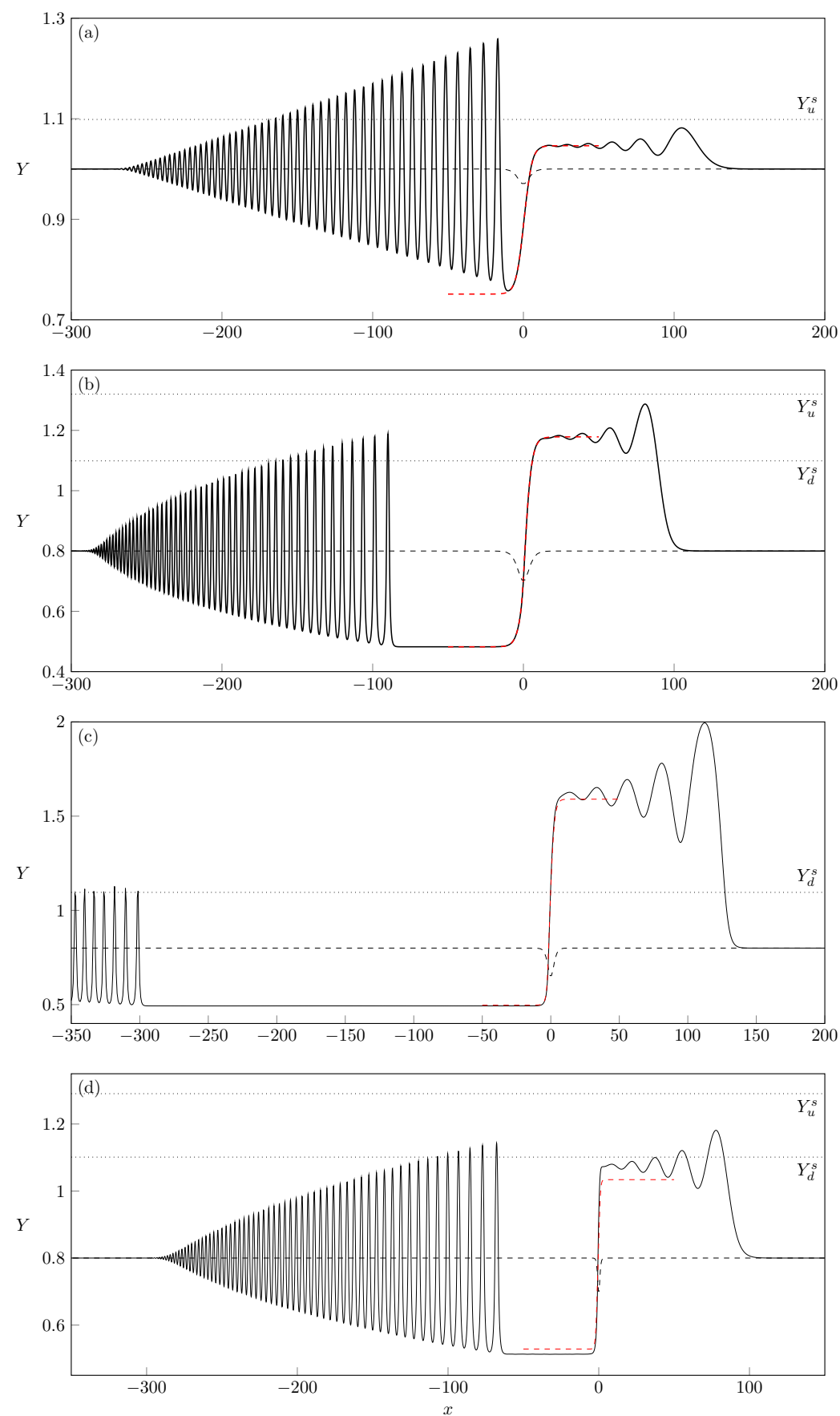

Figure 14: Contour dynamic simulations showing critically-controlled flow in the full QG problem. In (a), the downstream DSW is attached to the topography, in (c) the upstream DSW is attached, and in (b) and (d) both DSWs are detached. Red dashed curves show the dispersive controlled solution, black dashed curves show the topography, and black dotted lines show the solitary-wave amplitude predictions from dispersive shock-fitting. Full details are given in table 3. 

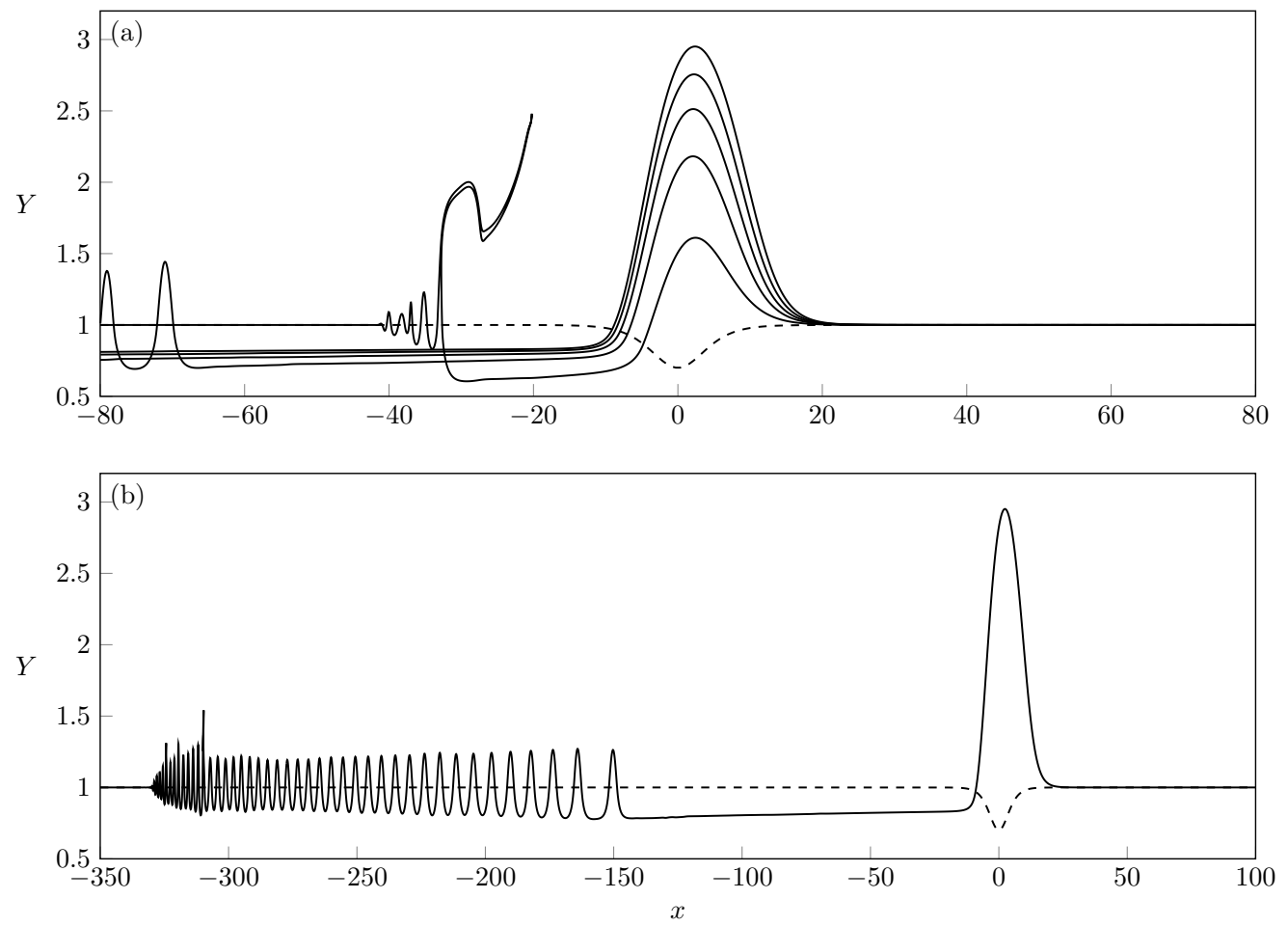

Figure 15: Contour dynamic simulation in the offshore plume regime. (a) Snapshots of the solution in the source region, every 200 time units starting from $t=100$. (b) The solution at $t=900$. Full details are given in table 3 .

\begin{tabular}{c|ccccccc} 
Subplot & Type & $F$ & $Y_{0}$ & $\Delta$ & $a$ & $\epsilon$ & $t$ \\
\hline & & & & & & & \\
$14(\mathrm{a})$ & Downstream attached & 0.75 & 1 & 0.03 & 1.07 & 0.2 & 900 \\
$14(\mathrm{~b})$ & Both detached & 0.8 & 0.8 & 0.1 & 1.19 & 0.2 & 700 \\
$14(\mathrm{c})$ & Upstream attached & 0.92 & 0.8 & 0.15 & 1.11 & 0.33 & 1900 \\
$14(\mathrm{~d})$ & Both detached & 0.8 & 0.7 & 0.1 & 1.2 & 1 & 700 \\
$15(\mathrm{~b})$ & Offshore plume & 1.1 & 1 & 0.3 & 0.88 & 0.2 & 900
\end{tabular}

Table 3: Details of the initial value problems displayed in figures 14 and 15 .

the dispersive long-wave theory provides an accurate quantitative description of the QG system up to $\epsilon \approx 0.5$.

Figure 15 shows a contour dynamic simulation in the offshore plume regime, where neither the controlled nor the supercritical solution exist and the shelf water is directed offshore. The growing behaviour is highlighted in (a), which shows snapshots of the solution in the source region, taken every 200 time units starting from $t=100$. Since the flow is unsteady the downstream state $Y_{\mathrm{d}}$ is not well-defined and the downstream waves are irregular and not ordered by amplitude (as seen in (b), where the full solution is shown at $t=900)$. The early-time development of the plume ejects a filament of coastal water into the open ocean (near $x=-40$ in (a)). Filamentation is a common feature 
of the contour-dynamic simulations, but is not shown in the other (late time) solutions presented here as the contour dynamics algorithm removes vortex patches below a certain size threshold for reasons of computational efficiency. (Small patches of vorticity do not contribute much to the dynamics but complex filament shapes require a lot of nodes and therefore take up a lot of computational time (Dritschel 1988).) The filamentation instability is due to a convergence in the velocity field (Stern 1986) and is one possible mechanism for the characteristic 'squirts' seen in the California Current system (Strub et al. 1991).

\section{Discussion}

A fully-nonlinear, dispersive long-wave model has been used to study hydraulic control of barotropic topographic Rossby waves. This model therefore complements previous works by Gill (1977) and Dale \& Barth (2001) by exploring control by coastal-trapped waves in the limit of small $S$, and extends the rigid-lid channel-flow model of Haynes et al. (1993) to a coastal setup. Section 3 classifies the behaviour of the hydraulic (nondispersive long-wave) equation and derives conditions for critical control in terms of $Y_{0}$, the far-field width of the shelfbreak; $\Delta$, the maximum magnitude of the shelfbreak perturbation; and the Froude number $F$. The downstream transition between the controlled state and the far-field flow is always resolved by a shock, while the upstream transition may be resolved by a shock, a rarefaction, or a compound-wave shock-rarefaction. Figure 7 gives an example of each type of resolution, and shows how the $(\Delta, F)$-plane is divided when $Y_{0}=0.8$. In $\S 4.2$ we use dispersive shock-fitting to analyse the dispersive long-wave equation, and show that shocks are replaced by modulated wave-trains which remain attached to the topography when $F$ is near the boundary for critical flow. Figure 14 confirms that this behaviour also occurs in the full QG system, and that the dispersive long-wave theory accurately predicts the solution in the forcing region and upstream at large times.

The present model is too simple to make quantitative comparisons with real CSWs. A sloping shelf and a more realistic background current can be incorporated following the discussion of $\S 2$, while other factors such as stratification, external forcing and dissipation will of course also be important in the real ocean. Further, in the quasi-geostrophic model we require that variations in fluid depth are small $(b \ll H)$ and that the inertial terms in the momentum equations are negligible compared to the rotation terms, so that vortical effects dominate the trajectory of a water column. However we note that the problem of a coastal outflow with uniform PV has been studied using a very similar model to that discussed here in both the quasi-geostrophic and long-wave shallow-water limits (Johnson et al. (2017) and Jamshidi \& Johnson (2019) respectively) with little change in the behaviour. Thus some of the qualitative features that are noted here merit further investigation. First and most important is to understand the regimes in which CTWs exert hydraulic control in the real ocean. Zhang \& Lentz (2017) and Saldías \& Allen (2020) both present numerical simulations of CTWs in a configuration very similar to that used here, albeit with sloping topography and a background flow driven by (constant) wind forcing. While ZL17 report steady flow when the background current opposes CTW propagation, and a response consistent with an arrested CTW (their figure 14), Saldías \& Allen (2020) find that their simulations never become steady in the forcing region and instead develop a meandering wave-train upstream. The reason for this difference is not clear, although Saldías \& Allen (2020) estimate that the Froude number for the first three CTW modes in their model is 2, 0.2 and 0.1 respectively so that they may be outside the range in which hydraulic control occurs. By exploring a wider range 
of flow speeds in a numerical model, one could potentially identify the boundaries for critical flow, as well as boundaries at which control changes between different modes. An improved understanding of hydraulic control could lead to a better parameterisation of boundary currents in global ocean models. Controlled flow decreases the transport of the background flow by recirculating some of the shelf water. This suggests that the parameterisation of such currents may need to account for large variations in shelf width that could significantly alter their structure and transport. Further, critically-controlled coastal flows may be associated with enhanced exchange between the shelf and the open ocean because they disturb the flow field far from the location of the perturbation. In the present model the PV front also marks the boundary between shelf and open-ocean water and so the large displacements in $Y$ seen in, for example, figure 7(e), correspond to large volumes of shelf water crossing the shelfbreak (upstream of the perturbation) and deep water mounting the shelf (downstream). Indeed, ZL17 show that criticallycontrolled flow leads to onshore velocities in their model of the Hudson Shelf Valley that are significantly stronger than the equivalent offshore velocity when the background flow is in the opposite direction, with a corresponding increase in transport (Zhang \& Lentz 2018, figure 3c).

Another interesting question concerns the formation of attached and detached DSWs. Figure 1 shows that the downstream DSW in ZL17 remains attached to the topography, while the present model suggests that this only occurs when the flow is very close to the subcritical boundary. Attached DSWs continually generate waves at one edge of the forcing region, and thus would be easier to identify and analyse in more complex models than detached DSWs, which may quickly degrade due to diabatic effects.

The 'off-shore plume' regime discussed in $\S 3.2$ and figure 15 is also of potential importance. Off-shore plumes occur for sufficiently large shelfbreak perturbations when the background current dominates over the alongshore flow generated by stretching of fluid columns that cross the shelfbreak. Instead of turning to propagate upstream, shelf water heads directly offshore and the flow never becomes steady in the forcing region. Topographic features are known to cause the displacement of boundary currents that flow counter to Rossby-wave propagation (for example, in the the separation of the Gulf Stream at Cape Hatteras (Tansley \& Marshall 2000) and the generation of upwelling filaments at Cape Ghir (Troupin et al. 2012)) and this may be explained by PV-conserving arguments similar to those proposed here. However the situation in the real ocean is more complicated than in the present flat-bottomed model, where columns of fluid that cross the shelfbreak can continue to move offshore easily. In an ocean with a sloping bottom, the proclivity of depth-integrated flow to follow isobaths limits exchange between the shelf and open ocean. Columns of fluid must instead separate from the bottom as they cross the shelfbreak, and thus the dynamics differ from the barotropic model employed here.

Perhaps the most restrictive assumption of the present model is that the assumption of piecewise-constant PV eliminates all but a single Rossby mode. The extension of control theory to flows with arbitrary PV distributions and therefore several modes is an important area of study, although Hughes (1985) shows that analytically identifying the controlling mode for a given geometry is likely to be difficult. Chapter 2.9 of Pratt \& Whitehead (2008) gives an overview of the barriers to analytic results in systems with non-uniform PV (albeit in the context of gravity-wave control) and further discussion of the consequences of this restriction. A different approach to studying hydraulic control in flows with arbitrary PV distributions is to follow Mitsudera \& Grimshaw (1990) and ZL17 and identify the controlling mode as that with the phase speed which is nearest to the (negative of the) background flow velocity. This suggests that control may in fact be 
more common in models with several modes, as there is a wider range of phase speeds that can become arrested. Grimshaw (1987) shows that the controlling mode is resonant, and thus if that mode has a non-zero coefficient initially it will grow to dominate the response at later times. However, in general mode-mode interactions may limit growth of resonant terms at higher modes, and thus control is likely to be restricted to the first few modes. Indeed, mode-1 resonant CSWs (defined as having small group velocity) have been observed off the coasts of Scotland (Gordon \& Huthnance 1987) and Antarctica (Wåhlin et al. 2016).

Finally, it is interesting that the agreement between dispersive long-wave theory and the QG system is not as strong as in the flat-bottom model of JJ20. In particular, the theoretical prediction for the downstream solitary wave amplitude can have a qualitatively different dependence on $F$ as that observed in the numerical results (figure 13(a), dashdotted curves and triangles). We have suggested that this is due to a turning point in the observed solitary wave speed, but a more detailed investigation of the equation is needed to confirm this. The numerical results appear to show a DSW forming downstream, but it is not clear what the equivalent for a finite-amplitude wave-front is for a DSW that is degenerate at the solitary wave end, or indeed whether the minimum in $\widetilde{s}$ does correspond to degeneracy of the Whitham system. Finding an equation that displays this behaviour and has an integrable structure could lead to further developments in the theory of dispersive shock waves.

\section{Appendix A. Derivation of boundaries for critical control}

Here we derive expressions for the boundaries of critical control, $F_{ \pm}(\Delta)$, by treating $Q_{e}$ and $C$ as polynomials in $Z=\exp (-Y)$. The case where the front is off the shelf $(j=-1)$ is a quadratic in $Z$. However, when the front is on the shelf the corresponding polynomial is cubic and the form of the solution is not informative, so in this case we just present the equation to be solved.

First, suppose that the flow is critical. If the front lies off the shelf at the maximum constriction, then solving the criticality condition gives (3.7). We call this kind of flow, where the front is off-shelf at the control point, off-shelf controlled. In the alternative situation, where the flow is on-shelf controlled, $Y$ is found by solving the following cubic equation in $Z$,

$$
-a^{2} Z^{3}+\left(\frac{a^{2}}{2} \mathrm{e}^{-Y_{T}}-1\right) Z^{2}+\frac{a^{2}}{2} \mathrm{e}^{-Y_{T}}=0,
$$

which has at most one root in $Z>0$. (The cubic polynomial $f(Z)$ defined by (A 1 ) is positive when $Z=0$, and either $Z=0$ is a local minimum or $f^{\prime}(Z)<0$ for all $Z>0$.) The transition between (3.7) and (A 1) occurs when

$$
\Delta=Y_{1}=Y_{0}-\log \left(\frac{1+\sqrt{1+a^{4}}}{a^{2}}\right),
$$

with off-shelf control occurring for $\Delta>Y_{1}$.

To find $F_{ \pm}(\Delta)$, we solve the criticality condition (3.3) and the condition for noncritical steady flow (3.2) simultaneously. For each pair $\left(Y_{0}, \Delta\right)$ this gives two values of $a$ (equivalently the Froude number $F$ ) at which the flow transitions from being non-critical to critical. For off-shelf control,

$$
a^{2}=2 \frac{Z_{T}^{3}-2 Z_{0} Z_{T}^{2}+Z_{T}-2 Z_{T}^{2}\left[2 Z_{0}\left(\cosh \left(Y_{0}\right)-\cosh \left(Y_{T}\right)\right)\right]^{1 / 2}}{\left(Z_{T}^{2}-1\right)^{2}},
$$


where we have introduced $Z_{T}=\exp \left(\Delta-Y_{0}\right)$ and $Z_{0}=\exp \left(-Y_{0}\right)$. For fixed $Y_{0}$, equation (A $3 a$ ) is used to give the supercritical boundary for controlled flow, $F_{+}(\Delta)$. For on-shelf control,

$$
a^{2}=\frac{2\left(Z_{0}-Z\right)}{\left(1-Z^{2}\right)\left(Z_{T} / Z-1\right)},
$$

where $Y$ is given by the solution to (A 1$)$. This gives the subcritical boundary $F_{-}(\Delta)$.

For wide shelves $\left(Z_{0}<1 / 2\right)$, the supercritical boundary $F_{+}(\Delta)$ is non-monotonic with a maximum at $\Delta_{0}$. Offshore plumes occur when $\Delta>\Delta_{0}$ and $F_{\mathrm{G}}(\Delta)<F<F_{\max }$, where

$$
\begin{aligned}
F_{\max } & =\frac{1}{1-Z_{0}^{2}}, \\
F_{\mathrm{G}}(\Delta) & =\frac{\cosh \left(Y_{0}-\Delta\right)}{\sinh \left(Y_{0}\right)} .
\end{aligned}
$$

For narrow shelves the maximum Froude number for critically-controlled solutions is

$$
F_{\max }=\frac{4 Z_{0}}{1+Z_{0}},
$$

which occurs at $\Delta=Y_{0}$.

\section{Appendix B. Conditions for dispersive shock-fitting}

El (2005) shows that one may extract the key observables of DSWs arising in the Riemann problem for a given equation providing certain conditions are satisfied. Thus, assuming that DSWs form outside of the topographic forcing region, we apply the following checks to $(2.22)$ with $Y_{h} \equiv Y_{0}$ :

(i) The equation admits a hydraulic limit obtained by introducing the slow variables $X=\epsilon x$ and $T=\epsilon t$. This is equation (2.16).

(ii) The linear dispersion relation is real-valued (c.f. equation (4.4)).

(iii) The system possesses at least two conservation laws. These are equations (2.22) and (2.23).

(iv) The equation supports periodic travelling-wave solutions, parameterised by three independent variables. These can be taken to be the constants of integration $s$, $\alpha$ and $E$ in the potential (4.7). The potential function must exhibit quadratic behaviour in the linear and solitary-wave limits, which is true for the present model provided $Y_{\infty}$ is sufficiently far from the coast that $\mathcal{G}=O(1)$.

(v) The Whitham system composed of the two period-averaged conservation laws plus the wavenumber conservation law $k_{t}+\omega_{x}=0$ is hyperbolic. This is required to ensure modulational stability of the wavetrain, and is easiest to check via numerical simulations. However, non-convexity of the flux function $Q_{e}$ implies that (2.22) is not genuinely nonlinear in an interval containing $Y_{2}$ and in many cases this leads to non-strict hyperbolicity (El et al. 2017). The compound-wave structures discussed in $\S 3$ carry over to the Whitham equations, and lead to richer behaviour than in the simple-wave case where the solution is a DSW. For transitions that cross the inflexion point $Y_{2}$, dispersive shock-fitting may be applied to the secondary Riemann problem with $Y_{ \pm}>Y_{2}$.

With these caveats about non-convexity and distance from the coast in mind, the key parameters may be extracted as follows. The wavenumber and conjugate wavenumber 
at the linear and solitary-wave edges of the DSW are found by solving the differential equations (c..f (El 2005))

$$
\begin{aligned}
\frac{\mathrm{d} k}{\mathrm{~d} Y} & =\frac{\partial \omega / \partial Y}{C(Y)-\partial \omega / \partial k}, \\
\frac{\mathrm{d} \widetilde{k}}{\mathrm{~d} Y} & =\frac{\partial \widetilde{\omega} / \partial Y}{C(Y)-\partial \widetilde{\omega} / \partial \widetilde{k}},
\end{aligned}
$$

For the present model, the general solutions to (B 1) are

$$
\begin{aligned}
k^{2}(Y) & =\frac{2}{3 \mathcal{G}(Y)^{2 / 3}} \int^{Y} \frac{C^{\prime}(\xi)}{\mathcal{G}(\xi)^{1 / 3}} \mathrm{~d} \xi, \\
\tilde{k}^{2}(Y) & =\frac{-2}{3 \mathcal{G}(Y)^{2 / 3}} \int^{Y} \frac{C^{\prime}(\xi)}{\mathcal{G}(\xi)^{1 / 3}} \mathrm{~d} \xi,
\end{aligned}
$$

which are connected to the far-field solution by applying the boundary conditions $k=0$ at the soliton edge of the DSW where $Y=Y_{+}$, and $\widetilde{k}=0$ at the linear-wave end where $Y=Y_{-}$. Then, we evaluate (B 2) at $Y_{\mp}$ to obtain $k$ and $\widetilde{k}$ as in (4.8), (4.10).

Declaration of interests: The authors report no conflict of interests.

\section{REFERENCES}

BRINK, K. H. 1991 Coastal-trapped waves and wind-driven currents over the continental shelf. Annu. Rev. Fluid Mech. 23 (1), 389-412.

Dale, A. C. \& Barth, J. A. 2001 The hydraulics of an evolving upwelling jet flowing around a cape. J. Phys. Oceanogr. 31 (1), 226-243.

Dritschel, D. G. 1988 Contour surgery: a topological reconnection scheme for extended integrations using contour dynamics. J. Comput. Phys. 77 (1), 240-266.

EL, G. A. 2005 Resolution of a shock in hyperbolic systems modified by weak dispersion. Chaos $15(3), 037103$.

El, G. A., Grimshaw, R. H. J. \& Smyth, N. F. 2006 Unsteady undular bores in fully nonlinear shallow-water theory. Phys. Fluids 18 (2), 027104.

El, G. A., Grimshaw, R. H. J. \& Smyth, N. F. 2009 Transcritical shallow-water flow past topography: finite-amplitude theory. J. Fluid Mech. 640, 187.

El, G. A., Hoefer, M. A. \& Shearer, M. 2017 Dispersive and diffusive-dispersive shock waves for nonconvex conservation laws. SIAM Rev. 59 (1), 3-61.

GiLl, A. E. 1977 The hydraulics of rotating-channel flow. J. Fluid Mech. 80 (04), 641-671.

Gill, A. E. \& Schumann, E. H. 1979 Topographically induced changes in the structure of an inertial coastal jet: application to the Agulhas Current. J. Phys. Oceanogr. 9 (5), 975-991.

Gordon, R. L. \& Huthnance, J. M. 1987 Storm-driven continental shelf waves over the Scottish continental shelf. Cont. Shelf Res. 7 (9), 1015-1048.

Grimshaw, R. H. J. 1987 Resonant forcing of barotropic coastally trapped waves. J. Phys. Oceanogr. 17 (1), 53-65.

Haynes, P. H., Johnson, E. R. \& Hurst, R. G. 1993 A simple model of Rossby-wave hydraulic behaviour. J. Fluid Mech. 253, 359-384.

Hughes, R. L. 1985 Multiple criticalities in coastal flows. Dyn. Atmos. Oceans 9 (4), 321-340.

JAMSHIDI, S. \& Johnson, E. R. 2019 Coastal outflow currents into a buoyant layer of arbitrary depth. J. Fluid Mech. 858, 656-688.

JAMShidi, S. \& Johnson, E. R. 2020 The long-wave potential-vorticity dynamics of coastal fronts. J. Fluid Mech. 888, A19.

Johnson, E. R. \& Clarke, S. R. 1999 Dispersive effects in Rossby-wave hydraulics. J. Fluid Mech. 401, 27-54.

Johnson, E. R. \& Clarke, S. R. 2001 Rossby wave hydraulics. Annu. Rev. Fluid Mech. 33 (1), 207-230. 
Johnson, E. R. \& McDonald, N. R. 2006 Vortical source-sink flow against a wall: The initial value problem and exact steady states. Phys. Fluids 18 (7), 076601.

Johnson, E. R., Southwick, O. R. \& McDonald, N. R. 2017 The long-wave vorticity dynamics of rotating buoyant outflows. J. Fluid Mech. 822, 418-443.

Kamchatnov, A. M. 2019 Dispersive shock wave theory for nonintegrable equations. Phys. Rev. E 99 (1), 012203.

KuBokAwA, A. 1991 On the behaviour of outflows with low potential vorticity from a sea strait. Tellus A 43 (2), 168-176.

Martell, C. M. \& Allen, J. S. 1979 The generation of continental shelf waves by alongshore variations in bottom topography. J. Phys. Oceanogr. 9 (4), 696-711.

Miller, A. J., Lermusiaux, P. F. J. \& Poulain, P.-M. 1996 A topographic-Rossby mode resonance over the Iceland-Faeroe Ridge. J. Phys. Oceanogr. 26 (12), 2735-2747.

Mitsudera, H. \& Grimshaw, R. H. J. 1990 Resonant forcing of coastally trapped waves in a continuously stratified ocean. Pure Appl. Geophys. 133 (4), 635-664.

Pratt, L. J. \& Stern, M. E. 1986 Dynamics of potential vorticity fronts and eddy detachment. J. Phys. Oceanogr. 16 (6), 1101-1120.

Pratt, L. J. \& Whitehead, J. A. 2008 Rotating Hydraulics, , vol. 1. Springer.

Saldías, G. S. \& Allen, S. E. 2020 The influence of a submarine canyon on the circulation and cross-shore exchanges around an upwelling front. J. Phys. Oceanogr. 50 (6), 1677-1698.

Stern, M. E. 1986 On the amplification of convergences in coastal currents and the formation of squirts. J. Mar. Res. 44 (3), 403-421.

Strub, P. T., Kosro, P. M. \& Huyer, A. 1991 The nature of the cold filaments in the California Current System. J. Geophys. Res. - Oceans 96 (C8), 14743-14768.

Tansley, C. E. \& Marshall, D. P. 2000 On the influence of bottom topography and the Deep Western Boundary Current on Gulf Stream separation. J. Mar. Res. 58 (2), 297-325.

Troupin, C., Mason, E., Beckers, J.-M. \& Sangrà, P. 2012 Generation of the Cape Ghir upwelling filament: A numerical study. Ocean Modell. 41, 1-15.

Wåhlin, A. K., Kalen, O., Assmann, K. M., Darelius, E., Ha, H. K., Kim, T.-W. \& LeE, S. H. 2016 Subinertial oscillations on the Amundsen Sea shelf, Antarctica. J. Phys. Oceanogr. 46 (9), 2573-2582.

Zhang, W. G. \& Lentz, S. J. 2017 Wind-driven circulation in a shelf valley. part I: Mechanism of the asymmetrical response to along-shelf winds in opposite directions. $J$. Phys. Oceanogr. 47 (12), 2927-2947.

Zhang, W. G. \& Lentz, S. J. 2018 Wind-driven circulation in a shelf valley. part II: Dynamics of the along-valley velocity and transport. J. Phys. Oceanogr. 48 (4), 883-904. 